\title{
Characterization of the traces on the boundary of functions in magnetic Sobolev spaces
}

\author{
Hoai-Minh Nguyen ${ }^{\mathrm{a}}$, Jean Van Schaftingen ${ }^{\mathrm{b}, *, 1}$ \\ a École Polytechnique Fédérale de Lausanne (EPFL), SB, CAMA, Station 8, \\ CH-1015 Lausanne, Switzerland \\ b Université catholique de Louvain (UCLouvain), Institut de Recherche \\ en Mathématique et Physique, Chemin du Cyclotron 2 bte L7.01.01, \\ 1348 Louvain-la-Neuve, Belgium
}

\section{A R T I C L E I N F O}

\section{Article history:}

Received 6 May 2019

Received in revised form 5 February 2020

Accepted 14 May 2020

Available online 8 June 2020

Communicated by Tristan Rivière

\section{$M S C$ :}

$46 \mathrm{E} 35$

26A33

$35 \mathrm{Q} 40$

$78 \mathrm{~A} 25$

$82 \mathrm{D} 40$

\section{Keywords:}

Fractional magnetic Sobolev spaces

Trace theory

Extension theorems

Interpolation of Banach spaces

\begin{abstract}
A B S T R A C T
We characterize the trace of magnetic Sobolev spaces defined in a half-space or in a smooth bounded domain in which the magnetic field $A$ is differentiable and its exterior derivative corresponding to the magnetic field $d A$ is bounded. In particular, we prove that, for $d \geq 1$ and $p>1$, the trace of the magnetic Sobolev space $W_{A}^{1, p}\left(\mathbb{R}_{+}^{d+1}\right)$ is exactly $W_{A^{\prime \prime}}^{1-1 / p, p}\left(\mathbb{R}^{d}\right)$ where $A^{\prime \prime}(x)=\left(A_{1}, \ldots, A_{d}\right)(x, 0)$ for $x \in \mathbb{R}^{d}$ with the convention $A=\left(A_{1}, \ldots, A_{d+1}\right)$ when $A \in C^{1}\left(\overline{\mathbb{R}_{+}^{d+1}}, \mathbb{R}^{d+1}\right)$. We also characterize fractional magnetic Sobolev spaces as interpolation spaces and give extension theorems from a halfspace to the entire space.
\end{abstract}

() 2020 Elsevier Inc. All rights reserved.

\footnotetext{
* Corresponding author.

E-mail addresses: hoai-minh.nguyen@epfl.ch (H.-M. Nguyen), Jean.VanSchaftingen@uclouvain.be (J. Van Schaftingen).

1 J. Van Schaftingen was partially supported by the Projet de Recherche (Fonds de la Recherche Scientifique - FNRS) No. T.1110.14 "Existence and asymptotic behavior of solutions to systems of semilinear elliptic partial differential equations". J. Van Schaftingen acknowledges the hospitality of the EPFL where a substantial part of this work was performed.
} 


\section{Introduction}

The first-order magnetic Sobolev space $W_{A}^{1, p}(\Omega)$ on a given open set $\Omega \subseteq \mathbb{R}^{d+1}$ with $d \geq 1$ is defined, for a given exponent $p \in[1,+\infty)$, a vector field $A \in C^{1}\left(\Omega, \mathbb{R}^{d+1}\right)$, as $[3,4,6,11,16,19,29],[31, \S 1.1]$

$$
W_{A}^{1, p}(\Omega) \triangleq\left\{U \in W_{\mathrm{loc}}^{1,1}(\Omega, \mathbb{C}) ;\|U\|_{W_{A}^{1, p}(\Omega)}^{p} \triangleq \int_{\Omega}|U|^{p}+\left|\nabla_{A} U\right|^{p}<+\infty\right\},
$$

where the weak covariant gradient $\nabla_{A} U$ associated with $A$ of $U \in W_{\text {loc }}^{1,1}(\Omega, \mathbb{C})$ is defined $\mathrm{as}^{2}$

$$
\nabla_{A} U \triangleq \nabla U+i A U \quad \text { in } \Omega
$$

Magnetic Sobolev spaces arise naturally for $p=2$ and $d=2$ (corresponding to $\Omega \subseteq \mathbb{R}^{3}$ ) in quantum mechanics in the presence of a magnetic field described through its magnetic vector potential $A \in C^{1}\left(\Omega, \mathbb{R}^{3}\right)$; the function $U: \Omega \rightarrow \mathbb{C}$ is then a wavefunction and the integral in (1.1) is the quadratic form associated to the quantum mechanical Hamiltonian of a particle in a magnetic field (see, e.g., [14, Chapter 16], [17, Chapter XV]). In physical models, the only observable quantities are the magnetic field $B=\nabla \times A \simeq d A \in C\left(\Omega, \bigwedge^{2} \mathbb{R}^{3}\right)$ and the probability density $|U|^{2}$. Here and in what follows, $d A$ denotes the exterior derivative of $A$; for this, we consider $A$ as a differential form in $C^{1}\left(\Omega, \bigwedge^{1} \mathbb{R}^{d+1}\right)$. The prevalent role of the magnetic field and of the probability density is reflected by the gauge invariance invariance of the model: the invariance of the Hamiltonian quadratic form defined by the right-hand side (1.1) under a change of variables $A \mapsto A+\nabla \Phi$ and $U \mapsto e^{-i \Phi} U$, for any phase shift $\Phi \in C^{1}(\Omega, \mathbb{R})$, see, e.g., [19, chapter 7]. Geometrically, the invariant quantity $i d A$ is the curvature of the associated $U(1)$-connection (see for example [32, Chapter 11]).

Magnetic Sobolev spaces $W_{A}^{1, p}(\Omega)$ generalize classical Sobolev spaces $W^{1, p}(\Omega)$, in which $A \equiv 0$, defined by

$$
W^{1, p}(\Omega) \triangleq\left\{U \in L^{p}(\Omega) ;\|U\|_{W^{1, p}(\Omega)}^{p} \triangleq \int_{\Omega}|U|^{p}+|\nabla U|^{p}<+\infty\right\} .
$$

\footnotetext{
${ }^{2}$ Two opposite conventions are in use for the sign of the second term in (1.2), we follow [3,11,19,29], and we have thus opposite convention to $[4,6,16],[31, \S 1.1]$. The presence of two opposite conventions is related to charge of the particles that are studied; both conventions are equivalent up to complex conjugation.
} 
For $0<s<1$ and $1 \leq p<+\infty$, the fractional Sobolev (Sobolev-Slobodecki乞) space is defined as

$$
W^{s, p}(\partial \Omega) \triangleq\left\{u \in L^{p}(\partial \Omega, \mathbb{C}) ;\|u\|_{W^{s, p}(\partial \Omega)}^{p} \triangleq\|u\|_{L^{p}(\partial \Omega)}^{p}+|u|_{W^{s, p}(\partial \Omega)}^{p}<+\infty\right\}
$$

where the Gagliardo seminorm $|u|_{W^{s, p}(\partial \Omega)}$ of the measurable function $u: \partial \Omega \rightarrow \mathbb{C}$ is given by

$$
|u|_{W^{s, p}(\partial \Omega)}^{p} \triangleq \iint_{\partial \Omega \times \partial \Omega} \frac{|u(y)-u(x)|^{p}}{|y-x|^{d+s p}} \mathrm{~d} x \mathrm{~d} y .
$$

When the set $\Omega$ is bounded and its boundary is of class $C^{1}$, or $\Omega=\mathbb{R}_{+}^{d+1} \triangleq\{(x, t) \in$ $\left.\mathbb{R}^{d} \times \mathbb{R} ; t>0\right\}$, and when $p>1$, the trace theory is well known since Gagliardo's pioneering work [13] (see also [9, §10.17-10.18 and Proposition 17.1], [22,30,36]). The trace linear operator Tr defined by

$$
\begin{aligned}
\operatorname{Tr}: C^{1}(\bar{\Omega}) & \rightarrow C^{1}(\partial \Omega) \\
U & \mapsto \operatorname{Tr}=\left.U\right|_{\partial \Omega},
\end{aligned}
$$

satisfies, for some positive constant $C_{p, \Omega}$ and for every $U \in C^{1}(\Omega)$, the estimate

$$
\|\operatorname{Tr} U\|_{W^{1-1 / p, p}(\partial \Omega)} \leq C_{p, \Omega}\|U\|_{W^{1, p}(\Omega)},
$$

and therefore, the linear operator $\operatorname{Tr}$ extends to a bounded linear map from the Sobolev space $W^{1, p}(\Omega)$ into fractional Sobolev space $W^{1-1 / p, p}(\partial \Omega)$. Conversely, there exists a bounded linear operator Ext : $W^{1-1 / p, p}(\partial \Omega) \rightarrow W^{1, p}(\Omega)$ such that for any $u \in W^{1-1 / p, p}(\partial \Omega)$,

$$
\operatorname{Tr}(\operatorname{Ext} u)=u \text { on } \partial \Omega \quad \text { and } \quad\|\operatorname{Ext} u\|_{W^{1, p}(\Omega)} \leq C_{p, \Omega}^{\prime}\|u\|_{W^{1-1 / p, p}(\partial \Omega)},
$$

for some positive constant $C_{p, \Omega}^{\prime}$ independent of $u$. In particular, the map $\operatorname{Tr}$ is surjective. Consequently, the image under the trace operator of the space $W^{1, p}(\Omega)$ is exactly the space $W^{1-1 / p, p}(\partial \Omega)$. The space of traces can also be described as the real interpolations space $\left(L^{p}\left(\mathbb{R}^{d}\right), W^{1, p}\left(\mathbb{R}^{d}\right)\right)_{1-1 / p, p}$ in the framework of interpolation of Banach spaces [20, Théorème VI.2.1].

The trace theory for $W_{A}^{1, p}(\Omega)$ can be easily derived from the one of $W^{1, p}(\Omega)$ when the magnetic potential $A$ is bounded. In fact, by the triangle inequality,

$$
\left|\left\|\nabla_{A} u\right\|_{L^{p}(\Omega)}-\|\nabla u\|_{L^{p}(\Omega)}\right| \leq\|A\|_{L^{\infty}(\Omega)}\|u\|_{L^{p}(\Omega)},
$$

it follows that $W_{A}^{1, p}(\Omega)=W^{1, p}(\Omega)$ in this case. Hence the trace space of $W_{A}^{1, p}(\Omega)$ is the space $W^{1-1 / p, p}(\partial \Omega)$ as well. The situation becomes more delicate when $\Omega=\mathbb{R}_{+}^{d+1}$ and 
$A$ is not assumed to be bounded but its total derivative $D A$ or, even more physically, its exterior derivative $d A$ is bounded. This type of assumption on $A$ appears naturally in many problems in physics for which $A$ is linear in simple settings. Moreover, even when $A$ is bounded, the quantitative bounds resulting (1.3) depend on the uniform norm $\|A\|_{L^{\infty}(\Omega)}$ which is not gauge-invariant; it would be desirable to have estimates depending rather on $d A$. To our knowledge, a characterization of the trace of $W_{A}^{1, p}\left(\mathbb{R}_{+}^{d+1}\right)$ is not known under such assumption on $A$. The goal of this work is to give a complete answer to this question. Besides its own interest concerning boundary values in problems of calculus of variations and partial differential equations, this is closely related to classes of fractional magnetic problems motivated by relativistic magnetic quantum physical models [15] that have been studied recently [1,2,5,8,12,18,25-28,33,37].

Given $0<s<1,1 \leq p<+\infty$, and $A^{\prime \prime} \in C\left(\mathbb{R}^{d}, \mathbb{R}^{d}\right)$, we define, for any measurable function $u: \mathbb{R}^{d} \rightarrow \mathbb{C}$, the magnetic Gagliardo semi-norm

$$
|u|_{W_{A^{\prime \prime}}^{s, p}\left(\mathbb{R}^{d}, \mathbb{C}\right)}^{p} \triangleq \iint_{\mathbb{R}^{d} \times \mathbb{R}^{d}} \frac{\left|e^{i \mathcal{I}_{A^{\prime \prime}}(x, y)} u(y)-u(x)\right|^{p}}{|y-x|^{d+s p}} \mathrm{~d} x \mathrm{~d} y,
$$

where the potential $\mathcal{I}_{A^{\prime \prime}}: \mathbb{R}^{d} \times \mathbb{R}^{d} \rightarrow \mathbb{R}$ is defined for each $x, y \in \mathbb{R}^{d}$ by

$$
\mathcal{I}_{A^{\prime \prime}}(x, y) \triangleq \int_{0}^{1} A^{\prime \prime}((1-t) x+t y) \cdot(y-x) \mathrm{d} t .
$$

Here and in what follows - denotes the usual complex scalar product defined for $v=$ $\left(v_{1}, \ldots, v_{d}\right)$ and $w=\left(w_{1}, \ldots, w_{d}\right) \in \mathbb{C}^{d}$ by $v \cdot w=v_{1} \bar{w}_{1}+\cdots+v_{d} \bar{w}_{d}$. For a vector field $A=\left(A_{1}, \ldots, A_{d+1}\right) \in C\left(\overline{\mathbb{R}_{+}^{d+1}}, \mathbb{R}^{d+1}\right)$, we will consider $A$ 's parallel component on the boundary $A^{\prime \prime}: \mathbb{R}^{d} \rightarrow \mathbb{R}^{d}$ defined for each $x \in \mathbb{R}^{d}$ by

$$
A^{\prime \prime}(x) \triangleq\left(A_{1}, \ldots, A_{d}\right)(x, 0)
$$

Our first main result is

Theorem 1.1. Let $d \geq 1$ and $1<p<+\infty$. There exists a positive constant $C_{d, p}$ depending only on $d$ and $p$ such that if $A \in C^{1}\left(\overline{\mathbb{R}_{+}^{d+1}}, \mathbb{R}^{d+1}\right)$ and $\|d A\|_{L^{\infty}\left(\mathbb{R}_{+}^{d+1}\right)} \leq \beta$, then

(i) for each $U \in C_{c}^{1}\left(\overline{\mathbb{R}_{+}^{d+1}}, \mathbb{C}\right)$,

$$
\begin{aligned}
& |U(\cdot, 0)|_{W_{A^{\prime \prime}}^{1-1 / p, p}\left(\mathbb{R}^{d}\right)}+\beta^{\frac{1}{2}-\frac{1}{2 p}}\|U(\cdot, 0)\|_{L^{p}\left(\mathbb{R}^{d}\right)} \\
& \leq C_{d, p}\left(\left\|\nabla_{A} U\right\|_{L^{p}\left(\mathbb{R}_{+}^{d+1}\right)}+\beta^{\frac{1}{2}}\|U\|_{L^{p}\left(\mathbb{R}_{+}^{d+1}\right)}\right),
\end{aligned}
$$


(ii) for each $u \in C_{c}^{1}\left(\mathbb{R}^{d}, \mathbb{C}\right)$, there exists $U \in C_{c}^{1}\left(\overline{\mathbb{R}_{+}^{d+1}}\right)$ depending linearly on $u$ and depending on $\beta$ such that $U(x, 0)=u(x)$ in $\mathbb{R}^{d}$ and

$$
\left\|\nabla_{A} U\right\|_{L^{p}\left(\mathbb{R}_{+}^{d+1}\right)}+\beta^{\frac{1}{2}}\|U\|_{L^{p}\left(\mathbb{R}_{+}^{d+1}\right)} \leq C_{d, p}\left(|u|_{W_{A^{\prime \prime}}^{1-1 / p, p}\left(\mathbb{R}^{d}\right)}+\beta^{\frac{1}{2}-\frac{1}{2 p}}\|u\|_{L^{p}\left(\mathbb{R}^{d}\right)}\right) .
$$

The conclusions of Theorem 1.1 are gauge-invariant: all the functional norms are gauge-invariant and the constants only depend through $\beta$ which is an upper bound of the norm $\|d A\|_{L^{\infty}\left(\mathbb{R}_{+}^{d+1}\right)}$ of the magnetic field on the half-space.

As a consequence of Theorem 1.1, by a standard density argument (see Section 4), we obtain the following characterization of the trace of the space $W_{A}^{1, p}\left(\mathbb{R}_{+}^{d+1}\right)$ :

Theorem 1.2. Let $d \geq 1$ and $1<p<+\infty$. Assume that $A \in C^{1}\left(\overline{\mathbb{R}_{+}^{d+1}}, \mathbb{R}^{d+1}\right)$ and that $d A \in L^{\infty}\left(\mathbb{R}_{+}^{d+1}, \bigwedge^{2} \mathbb{R}^{d+1}\right)$. The trace mapping

$$
\begin{aligned}
\operatorname{Tr}: W_{A}^{1, p}\left(\mathbb{R}_{+}^{d+1}, \mathbb{C}\right) & \rightarrow W_{A^{\prime \prime}}^{1-1 / p, p}\left(\mathbb{R}^{d}, \mathbb{C}\right) \\
U\left(x, x_{d+1}\right) & \mapsto U(x, 0)
\end{aligned}
$$

is linear and continuous. There also exists a linear continuous mapping

$$
\text { Ext }: W_{A^{\prime \prime}}^{1-1 / p, p}\left(\mathbb{R}^{d}, \mathbb{C}\right) \rightarrow W_{A}^{1, p}\left(\mathbb{R}_{+}^{d+1}, \mathbb{C}\right)
$$

such that $\operatorname{Tr} \circ \operatorname{Ext}_{\mathbb{R}_{+}^{d+1}}$ is the identity on $W_{A^{\prime \prime}}^{1-1 / p, p}\left(\mathbb{R}^{d}\right)$. Moreover, the corresponding estimates of Theorem 1.1 with $u=\operatorname{Tr} U$ and $U=\operatorname{Ext} u$ are valid.

In the case where the magnetic field $d A$ is constant, we obtain the following improvements:

Theorem 1.3. Let $d \geq 1$ and $1<p<+\infty$. Assume that $A \in C^{1}\left(\overline{\mathbb{R}_{+}^{d+1}}, \mathbb{R}^{d+1}\right)$ and that $d A$ is constant. We have, with $u \triangleq \operatorname{Tr} U$,

$$
|u|_{W_{A^{\|}}^{1-1 / p, p}\left(\mathbb{R}^{d}\right)} \leq C_{d, p}\left\|\nabla_{A} U\right\|_{L^{p}\left(\mathbb{R}_{+}^{d+1}\right)} .
$$

Moreover, for every $u \in W_{A^{\prime \prime}}^{1, p}\left(\mathbb{R}^{d}\right)$, there exists $U \in W_{A}^{1, p}\left(\mathbb{R}_{+}^{d+1}\right)$ such that $\operatorname{Tr} U=u$ and

$$
\left\|\nabla_{A} U\right\|_{L^{p}\left(\mathbb{R}_{+}^{d+1}\right)}+\|d A\|^{\frac{1}{2}}\|U\|_{L^{p}\left(\mathbb{R}_{+}^{d+1}\right)} \leq C_{d, p}|u|_{W_{A^{\prime \prime}}^{1-1 / p, p}\left(\mathbb{R}^{d}\right)} .
$$

We later show that the space $W_{A^{\prime \prime}}^{s, p}\left(\mathbb{R}^{d}\right)$ with $0<s<1$ and $p \geq 1$ is the trace space of the space $W_{A, 1-(1-s) p}^{1, p}\left(\mathbb{R}^{d}\right)$ whose definition is given in (4.1); moreover, the corresponding estimates hold (see Theorems 4.3 and 5.4).

We establish similar estimates for a smooth bounded domain $\Omega \subset \mathbb{R}^{d+1}$ and a magnetic potential $A \in C^{1}\left(\bar{\Omega}, \mathbb{R}^{d+1}\right)$. It is worth noting that the trace theory in this 
setting is known as in the case $A \equiv 0$. Nevertheless, our estimates (Proposition 6.4 and Proposition 6.5) are gauge-invariant, and sharpen estimates in the semi-classical limit (Proposition 6.6).

As a consequence of the trace theorems, we derive a characterization of the space $W_{A^{\prime \prime}}^{s, p}\left(\mathbb{R}^{d}, \mathbb{C}\right.$ ) as an interpolation space (Theorem 7.2 ). We also observe that the characterization of traces is also independent on the side of the hyperplane from which the trace is taken or to which the extension is made (this fact is not completely trivial, see Remark 8.2). Consequently, the trace theorem provides an extension theorem from a half-space to the whole space (Theorem 8.1).

In an appendix, we show that under the assumption that some derivatives of the vector field $A$ are bounded, our magnetic fractional spaces have equivalent norms to other families of fractional spaces defined in the literature (Proposition A.1).

We now describe briefly the idea of the proof of the trace theory. The proofs of the trace estimates and of the construction of the extension are based on standard strategies that go back to Gagliardo's seminal work [13]. Concerning Theorem 1.1 and its variants (Propositions 2.1 and 3.1), the key point of our analysis lies on the observation that $A^{\prime \prime}$ defined in $\mathbb{R}^{d}$ by (1.5) encodes the information the trace space of $W_{A}^{1, p}\left(\mathbb{R}_{+}^{d+1}\right)$, and on an appropriate extension formula given in (3.1). The proof of the trace estimates also involves Stokes theorems (Lemma 2.2) and a simple, useful, observation given in Lemma 2.3. Concerning Theorem 1.3 and its variants (Theorem 5.4), the new part is the trace estimates (see, e.g., (1.6)). To this end, the Stokes formula and an averaging argument are used while taking into account the fact $d A$ is constant. The proof for a domain $\Omega$ uses the results in the half space via local charts.

\section{Trace estimate for bounded magnetic fields}

In this section, we prove the following trace estimate on the boundary of the half-space with a bounded magnetic field, which covers (i) in Theorem 1.1.

Proposition 2.1. Let $d \geq 1,0<s<1$, and $1 \leq p<+\infty$. There exists a positive constant $C_{d, s, p}$ depending only on $d, s$ and $p$ such that if $A \in C^{1}\left(\overline{\mathbb{R}_{+}^{d+1}}, \mathbb{R}^{d+1}\right)$, if $\|d A\|_{L^{\infty}\left(\mathbb{R}_{+}^{d+1}\right)} \leq$ $\beta$ and if $U \in C_{c}^{1}\left(\overline{\mathbb{R}_{+}^{d+1}}, \mathbb{C}\right)$, then

$$
|U(\cdot, 0)|_{W_{A^{\prime \prime}}^{s, p}\left(\mathbb{R}^{d}\right)}^{p} \leq C_{d, s, p} \underset{\mathbb{R}^{d} \times(0,+\infty)}{\iint_{\left(\left.\nabla_{A} U(z, t)\right|^{p}+\beta^{\frac{p}{2}}|U(z, t)|^{p}\right.}} \mathrm{d} z \mathrm{~d} t
$$

and

$$
\begin{aligned}
& \|U(\cdot, 0)\|_{L^{p}\left(\mathbb{R}^{d}\right)}^{p} \\
& \quad \leq C_{d, s, p}\left(\underset{\mathbb{R}^{d} \times(0,+\infty)}{\iint_{(1-(1-s) p}} \frac{\left|\nabla_{A} U(z, t)\right|^{p}}{t^{1-(1)}} \mathrm{d} t \mathrm{~d} z\right)^{1-s}\left(\iint_{\mathbb{R}^{d} \times(0,+\infty)} \frac{|U(z, t)|^{p}}{t^{1-(1-s) p}} \mathrm{~d} t \mathrm{~d} z\right)^{s} .
\end{aligned}
$$


As a consequence of (2.2) by Young's inequality, we have

$$
\beta^{\frac{s p}{2}}\|U(\cdot, 0)\|_{L^{p}\left(\mathbb{R}^{d}\right)}^{p} \leq C^{\prime} \iint_{\mathbb{R}^{d} \times(0,+\infty)} \frac{\left|\nabla_{A} U(z, t)\right|^{p}+\beta^{\frac{p}{2}}|U(z, t)|^{p}}{t^{1-(1-s) p}} \mathrm{~d} z \mathrm{~d} t
$$

We first present several lemmas used in the proof of Proposition 2.1, before going to the proof of Proposition 2.1 at the end of the section.

We define for each $X, Y \in \overline{\mathbb{R}_{+}^{d+1}}$, the homotopy operator

$$
\mathcal{I}_{A}(X, Y) \triangleq \int_{0}^{1} A((1-\sigma) X+\sigma Y) \cdot(Y-X) \mathrm{d} \sigma
$$

We observe that by the change of variable $\tau=\sigma t$,

$$
\begin{aligned}
\mathcal{I}_{A}(X,(1-t) X+t Y) & =\int_{0}^{1} A((1-\sigma) X+\sigma((1-t) X+t Y)) \cdot t(Y-X) \mathrm{d} \sigma \\
& =\int_{0}^{1} A((1-\sigma t) X+\sigma t Y) \cdot t(Y-X) \mathrm{d} \sigma \\
& =\int_{0}^{t} A((1-\tau) X+\tau Y) \cdot(Y-X) \mathrm{d} \tau,
\end{aligned}
$$

and thus

$$
\frac{\mathrm{d}}{\mathrm{d} t} \mathcal{I}_{A}(X,(1-t) X+t Y)=A((1-t) X+t Y)[Y-X]
$$

Hence,

$\frac{\mathrm{d}}{\mathrm{d} t} e^{i \mathcal{I}_{A}(X,(1-t) X+t Y)} U((1-t) X+t Y)=e^{i \mathcal{I}_{A}(X,(1-t) X+t Y)} \nabla_{A} U((1-t) X+t Y) \cdot(Y-X)$

and therefore, by the fundamental theorem of calculus, we have

$$
e^{i \mathcal{I}_{A}(X, Y)} U(Y)-U(X)=\int_{0}^{1} e^{i \mathcal{I}_{A}(X,(1-t) X+t Y)} \nabla_{A} U((1-t) X+t Y) \cdot(Y-X) \mathrm{d} t
$$

The following result will be used repeatedly in the present work. It is essentially a reformulation of Stokes theorem. 
Lemma 2.2. If $d \geq 1, A \in C^{1}\left(\overline{\mathbb{R}_{+}^{d+1}}, \mathbb{R}^{d+1}\right)$, then for every $X, Y, Z \in \overline{\mathbb{R}_{+}^{d+1}}$, we have $\mathcal{I}_{A}(X, Y)+\mathcal{I}_{A}(Y, Z)+\mathcal{I}_{A}(Z, X)=\int_{0}^{1} \int_{0}^{1-\sigma} d A((1-\rho-\sigma) X+\rho Y+\sigma Z)[Y-X, Z-X] \mathrm{d} \rho \mathrm{d} \sigma$.

Proof. We have if $\sigma, \rho \in[0,1]$ and $\sigma+\rho \leq 1$,

$$
\begin{aligned}
& d A((1-\rho-\sigma) X+\rho Y+\sigma Z)[Y-X, Z-X] \\
& \quad=\frac{\mathrm{d}}{\mathrm{d} \rho} A((1-\rho-\sigma) X+\rho Y+\sigma Z)[Z-X]-\frac{\mathrm{d}}{\mathrm{d} \sigma} A((1-\rho-\sigma) X+\rho Y+\sigma Z)[Y-X] .
\end{aligned}
$$

Integrating with respect to $\sigma, \rho \in[0,1]$ with $\sigma+\rho \leq 1$ yields

$$
\begin{aligned}
\int_{0}^{1} \int_{0}^{1-\sigma} d A((1-\rho-\sigma) X & +\rho Y+\sigma Z)[Y-X, Z-X] \mathrm{d} \rho \mathrm{d} \sigma \\
& =\int_{0}^{1} \int_{0}^{1-\sigma} \frac{\mathrm{d}}{\mathrm{d} \rho} A((1-\rho-\sigma) X+\rho Y+\sigma Z)[Z-X] \mathrm{d} \rho \mathrm{d} \sigma \\
& -\int_{0}^{1} \int_{0}^{1-\rho} \frac{\mathrm{d}}{\mathrm{d} \sigma} A((1-\rho-\sigma) X+\rho Y+\sigma Z)[Y-X] \mathrm{d} \sigma \mathrm{d} \rho .
\end{aligned}
$$

By the fundamental theorem of calculus, for every $\sigma \in[0,1]$ we have

$$
\begin{aligned}
\int_{0}^{1-\sigma} \frac{\mathrm{d}}{\mathrm{d} \rho} A((1-\rho-\sigma) X & +\rho Y+\sigma Z)[Z-X] \mathrm{d} \rho \\
& =A((1-\sigma) Y+\sigma Z)[Z-X]-A((1-\sigma) X+\sigma Z)[Z-X],
\end{aligned}
$$

and for every $\rho \in[0,1]$,

$$
\begin{aligned}
\int_{0}^{1-\rho} \frac{\mathrm{d}}{\mathrm{d} \sigma} A((1-\rho-\sigma) X & +\rho Y+\sigma Z)[Y-X] \mathrm{d} \sigma \\
& =A(\rho Y+(1-\rho) Z)[Y-X]-A((1-\rho) X+\rho Y)[Y-X] .
\end{aligned}
$$

By inserting (2.8), (2.9) and (2.7) and by applying suitable change of variables, we obtain 


$$
\begin{aligned}
& \int_{0}^{1} \int_{0}^{1-\sigma} d A((1-\rho-\sigma) X+\rho Y+\sigma Z)[Y-X, Z-X] \mathrm{d} \rho \mathrm{d} \sigma \\
& \quad=\int_{0}^{1} A((1-\sigma) Y+\sigma Z)[Z-X] \mathrm{d} \sigma-\int_{0}^{1} A(\rho X+(1-\rho) Z)[Z-X] \mathrm{d} \rho \\
& \quad-\int_{0}^{1} A((1-\sigma) Y+\sigma Z)[Y-X] \mathrm{d} \sigma+\int_{0}^{1} A((1-\rho) X+\rho Y)[Y-X] \mathrm{d} \rho \\
& =\mathcal{I}_{A}(Z, X)+\mathcal{I}_{A}(Y, Z)+\mathcal{I}_{A}(X, Y),
\end{aligned}
$$

in view of the definition in (2.4).

Using Lemma 2.2, we can establish the following simple result which is the key ingredient of the proof of Proposition 2.1.

Lemma 2.3. If $d \geq 1, U \in C^{1}\left(\overline{\mathbb{R}_{+}^{d+1}}, \mathbb{C}\right)$, and $A \in C^{1}\left(\overline{\mathbb{R}_{+}^{d+1}}, \mathbb{R}^{d+1}\right)$, then for every $X, Y, Z \in \overline{\mathbb{R}_{+}^{d+1}}$, we have

$$
\begin{aligned}
\left|e^{i \mathcal{I}_{A}(X, Y)} U(Y)-U(X)\right| \leq\left|e^{i \mathcal{I}_{A}(Z, Y)} U(Y)-U(Z)\right| & +\left|e^{i \mathcal{I}_{A}(Z, X)} U(X)-U(Z)\right| \\
& +|U(Z)| \min \left\{2, \frac{1}{2}\|d A\|_{L^{\infty}}|(X-Z) \wedge(Y-Z)|\right\} .
\end{aligned}
$$

Proof. Since

$$
\left|e^{i \mathcal{I}_{A}(X, Y)} U(Y)-e^{i\left(\mathcal{I}_{A}(X, Y)+\mathcal{I}_{A}(Y, Z)\right)} U(Z)\right|=\left|e^{i \mathcal{I}_{A}(Z, Y)} U(Y)-U(Z)\right|,
$$

and

$$
\left|U(X)-e^{i \mathcal{I}_{A}(X, Z)} U(Z)\right|=\left|e^{i \mathcal{I}_{A}(Z, X)} U(X)-U(Z)\right|,
$$

by the triangle inequality, we obtain

$$
\begin{aligned}
\left|e^{i \mathcal{I}_{A}(X, Y)} U(Y)-U(X)\right| \leq\left|e^{i \mathcal{I}_{A}(Z, Y)} U(Y)-U(Z)\right|+\left|e^{i \mathcal{I}_{A}(Z, X)} U(X)-U(Z)\right| \\
+\left|e^{i\left(\mathcal{I}_{A}(X, Y)+\mathcal{I}_{A}(Y, Z)\right)} U(Z)-e^{i \mathcal{I}_{A}(X, Z)} U(Z)\right|
\end{aligned}
$$

We observe that

$$
\left|e^{i\left(\mathcal{I}_{A}(X, Y)+\mathcal{I}_{A}(Y, Z)\right)} U(Z)-e^{i \mathcal{I}_{A}(X, Z)} U(Z)\right|=\left|e^{i\left(\mathcal{I}_{A}(X, Y)+\mathcal{I}_{A}(Y, Z)+\mathcal{I}_{A}(Z, X)\right)}-1\right||U(Z)|
$$

and conclude with the inequality $\left|e^{i t}-1\right|=2|\sin t / 2| \leq \min \{2,|t|\}$ and by Lemma 2.2 . 
Proof of Proposition 2.1. Applying Lemma 2.3, we have, for each $x, y \in \mathbb{R}^{d}$ and with the notations $x \simeq(x, 0) \in \mathbb{R}_{+}^{d+1}, y \simeq(y, 0) \in \mathbb{R}_{+}^{d+1}, Z=\left(\frac{x+y}{2},|y-x|\right)$ and $u=U(\cdot, 0)$,

$$
\begin{aligned}
& \left|e^{i \mathcal{I}_{A}(x, y)} u(y)-u(x)\right| \\
& \quad \leq\left|e^{i \mathcal{I}_{A}(Z, x)} U(x)-U(Z)\right|+\left|e^{i \mathcal{I}_{A}(Z, y)} U(y)-U(Z)\right|+|U(Z)|\|d A\|_{L^{\infty}}^{\frac{1}{2}}|y-x| .
\end{aligned}
$$

Using (2.6), we derive from (2.10) that

$$
\begin{aligned}
\left|e^{i \mathcal{I}_{A}(x, y)} u(y)-u(x)\right| \leq C_{1}(|y-x| & \int_{0}^{1}\left|\nabla_{A} U((1-t) x+t Z)\right| \mathrm{d} t \\
& \left.+|y-x| \int_{0}^{1}\left|\nabla_{A} U((1-t) y+t Z)\right| \mathrm{d} t+\beta^{\frac{1}{2}}|y-x||U(Z)|\right) .
\end{aligned}
$$

Here and in what follows in this proof, $C_{1}, C_{2}, \ldots$ denote positive constants depending only on $d, p$, and $s$.

Since $0<s<1$, using the fact, for a measurable function $f$ defined on $[0,1]$, by Hölder's inequality, that

$$
\left(\int_{0}^{1}|f(t)| \mathrm{d} t\right)^{p} \leq C_{2} \int_{0}^{1} t^{(1-s)(p-1)}|f(t)|^{p} \mathrm{~d} t
$$

we derive from (2.11) that, since $Z=\left(\frac{x+y}{2},|y-x|\right)$,

$$
\begin{aligned}
& \frac{\left|e^{i \mathcal{I}_{A^{\prime \prime}}(x, y)} U(y)-U(x)\right|^{p}}{|y-x|^{d+s p}} \\
& \leq C_{3}\left(\int_{0}^{1} t^{(1-s)(p-1)} \frac{\left|\nabla_{A} U\left(\left(1-\frac{t}{2}\right) x+\frac{t}{2} y, t|y-x|\right)\right|^{p}}{|y-x|^{d-(1-s) p}} \mathrm{~d} t\right. \\
& \left.\quad+\int_{0}^{1} t^{(1-s)(p-1)} \frac{\left.\mid \nabla_{A} U\left(\left(1-\frac{t}{2}\right) y+\frac{t}{2} x, t|y-x|\right)\right)\left.\right|^{p}}{|y-x|^{d-(1-s) p} \mathrm{~d} t}+\beta^{\frac{p}{2}} \frac{\left|U\left(\frac{x+y}{2},|y-x|\right)\right|^{p}}{|y-x|^{d-(1-s) p}}\right) .
\end{aligned}
$$

We now estimate the integral of the left-hand side of (2.12) with respect to $x$ and $y$ by estimating the integrals of the three terms on the right-hand side. For every $t \in(0,1)$, making the change of variable $\eta=\left(1-\frac{t}{2}\right) x+\frac{t}{2} y$ and $\xi=t(x-y)$, we obtain 


$$
\begin{aligned}
& t^{(1-s)(p-1)} \iiint_{\mathbb{R}^{d} \times \mathbb{R}^{d}} \frac{\left|\nabla_{A}\left(U\left(1-\frac{t}{2}\right) x+\frac{t}{2} y, t|y-x|\right)\right|^{p}}{|y-x|^{d-(1-s) p}} \mathrm{~d} x \mathrm{~d} y \\
& \quad=\frac{1}{t^{1-s}} \iiint_{\mathbb{R}^{d} \times \mathbb{R}^{d}} \frac{\left|\nabla_{A} U(\eta,|\xi|)\right|^{p}}{|\xi|^{d-(1-s) p}} \mathrm{~d} \eta \mathrm{d} \xi=\frac{\left|\mathbb{S}^{d-1}\right|}{t^{1-s}} \iint_{\mathbb{R}^{d} \times(0,+\infty)} \frac{\left|\nabla_{A} U(\eta, r)\right|^{p}}{r^{1-(1-s) p}} \mathrm{~d} \eta \mathrm{d} r .
\end{aligned}
$$

Since $0<s<1$, it follows that

$$
\begin{aligned}
& \iint_{\mathbb{R}^{d} \times \mathbb{R}^{d}} \int_{0}^{1} t^{(1-s)(p-1)} \frac{\left.\mid \nabla_{A}\left(U\left(1-\frac{t}{2}\right) x+\frac{t}{2} y, t|y-x|\right)\right)\left.\right|^{p}}{|y-x|^{d-(1-s) p}} \mathrm{~d} t \mathrm{~d} x \mathrm{~d} y \\
& \leq C_{4} \int \underset{\mathbb{R}^{d} \times(0,+\infty)}{\int} \frac{\left|\nabla_{A} U(x, r)\right|^{p}}{r^{1-(1-s) p}} \mathrm{~d} x \mathrm{~d} r .
\end{aligned}
$$

Similarly, we have

$$
\begin{aligned}
\iint_{\mathbb{R}^{d} \times \mathbb{R}^{d}} \int_{0}^{1} t^{(1-s)(p-1)} \frac{\left.\mid \nabla_{A}\left(U\left(1-\frac{t}{2}\right) y+\frac{t}{2} x, t|y-x|\right)\right)\left.\right|^{p}}{|y-x|^{d-(1-s) p}} \mathrm{~d} t \mathrm{~d} x \mathrm{~d} y \\
\quad \leq C_{4} \iint_{\mathbb{R}^{d} \times(0,+\infty)} \frac{\left|\nabla_{A} U(x, r)\right|^{p}}{r^{1-(1-s) p}} \mathrm{~d} x \mathrm{~d} r .
\end{aligned}
$$

Using the change of variable $\eta=\frac{x+y}{2}$ and $\xi=y-x$, and the polar coordinates, by the same way to obtain $(2.13)$, we also reach

$$
\iint_{\mathbb{R}^{d} \times \mathbb{R}^{d}} \frac{\left|U\left(\frac{x+y}{2},|y-x|\right)\right|^{p}}{|x-y|^{d-(1-s) p}} \mathrm{~d} x \mathrm{~d} y=\left|\mathbb{S}^{d-1}\right| \underset{\mathbb{R}^{d} \times(0,+\infty)}{\iint_{(1-(1-s) p}} \frac{|U(\eta, r)|^{p}}{r^{1-(1-\infty)}} \mathrm{d} \eta \mathrm{d} r .
$$

Combining (2.12), (2.14), (2.15), and (2.16) yields

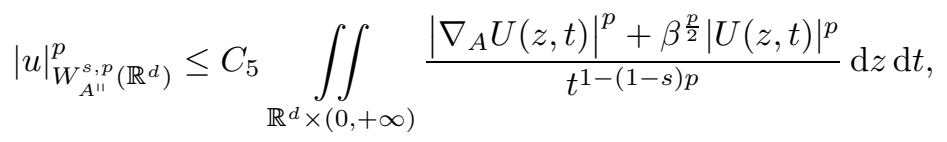

which is (2.1).

We next prove (2.2). Since, by the diamagnetic inequality $|\nabla| U|| \leq\left|\nabla_{A} U\right|$ everywhere in $\mathbb{R}_{+}^{d+1}$, we have, for each $x \in \mathbb{R}^{d}$ and $t \geq 0$,

$$
|U(x, 0)| \leq|U(x, t)|+\int_{0}^{t}\left|\nabla_{A} U(x, \tau)\right| \mathrm{d} \tau .
$$

It follows that, for $x \in \mathbb{R}^{d}$ and $\lambda>0$, 


$$
|U(x, 0)| \leq \int_{0}^{\lambda}\left|\nabla_{A} U(x, t)\right| \mathrm{d} t+\frac{2}{\lambda} \int_{\lambda / 2}^{\lambda}|U(x, t)| \mathrm{d} t .
$$

Using Hölder's inequality, we deduce since $0<s<1$ that

$$
|U(x, 0)|^{p} \leq C_{6} \lambda^{s p} \int_{0}^{+\infty} \frac{\left|\nabla_{A} U(x, t)\right|^{p}}{t^{1-(1-s) p}} \mathrm{~d} t+\frac{C_{7}}{\lambda^{(1-s) p}} \int_{0}^{+\infty} \frac{|U(x, t)|^{p}}{t^{1-(1-s) p}} \mathrm{~d} t
$$

and therefore by integration

$$
\begin{aligned}
& \int_{\mathbb{R}^{d}}|U(x, 0)|^{p} \mathrm{~d} x \\
& \quad \leq C_{6} \lambda^{s p} \underset{\mathbb{R}^{d} \times(0,+\infty)}{\iint_{0^{1-(1-s) p}}} \frac{\left|\nabla_{A} U(x, t)\right|^{p}}{t^{1-\infty}} \mathrm{d} x \mathrm{~d} t+\frac{C_{7}}{\lambda^{(1-s) p}} \iint_{\mathbb{R}^{d} \times(0,+\infty)} \frac{|U(x, t)|^{p}}{t^{1-(1-s) p}} \mathrm{~d} x \mathrm{~d} t .
\end{aligned}
$$

Optimizing with respect to $\lambda>0$, we obtain

$$
\begin{aligned}
& \int_{\mathbb{R}^{d}}|U(x, 0)|^{p} \mathrm{~d} x \\
& \quad \leq C_{8}\left(\underset{\mathbb{R}^{d} \times(0,+\infty)}{\iint_{t^{1-(1-s) p}}} \frac{\left|\nabla_{A} U(x, t)\right|^{p}}{\left.t^{1-(1-\infty} x \mathrm{~d} t\right)^{1-s}\left(\iint_{\mathbb{R}^{d} \times(0,+\infty)} \frac{|U(x, t)|^{p}}{t^{1-(1-s) p}} \mathrm{~d} x \mathrm{~d} t\right)^{s},}\right.
\end{aligned}
$$

which is $(2.2)$.

Using the same arguments, we obtain a localized version of Proposition 2.1 which will be used in Section 6 . In what follows $B(x, R)$ denotes the open ball in $\mathbb{R}^{d}$ centered at $x$ and of radius $R$; when $x=0$, we use the notation $B_{R}$ instead.

Proposition 2.4. Let $d \geq 1,0<s<1$ and $1 \leq p<+\infty$. There exists a positive constant $C_{d, s, p}$ depending only on $s, p$, and $d$ such that if $R \in(0,+\infty), A \in C^{1}\left(B_{R} \times[0, R], \mathbb{R}^{d+1}\right)$, if $\|d A\|_{L^{\infty}} \leq \beta$ and if $U \in C^{1}\left(B_{R} \times[0, R], \mathbb{C}\right)$ and $u=U(\cdot, 0)$, then

$$
\begin{aligned}
\iint_{B_{R} \times B_{R}} \frac{\left|e^{i \mathcal{I}_{A^{\prime \prime}}(x, y)} U(y, 0)-U(x, 0)\right|^{p}}{|y-x|^{d+s p}} \mathrm{~d} x & \mathrm{~d} y \\
& \leq C_{d, s, p} \iint_{B_{R} \times[0, R]} \frac{\left|\nabla_{A} U(z, t)\right|^{p}+\beta^{\frac{p}{2}}|U(z, t)|^{p}}{t^{1-(1-s) p} \mathrm{~d} t \mathrm{~d} z .}
\end{aligned}
$$




\section{Extension to the half-space}

In this section, we prove the following extension result which implies (ii) of Theorem 1.1 .

Proposition 3.1. Let $d \geq 1,0<s<1$, and $1 \leq p<+\infty$. There exists a positive constant $C_{d, s, p}$ depending only on $d, s$, and $p$ such that for every $A \in C^{1}\left(\overline{\mathbb{R}_{+}^{d+1}}, \mathbb{R}^{d+1}\right)$ with $\|d A\|_{L^{\infty}\left(\mathbb{R}_{+}^{d+1}\right)} \leq \beta$ and for any $u \in C_{c}^{1}\left(\mathbb{R}^{d}, \mathbb{C}\right)$ with compact support, one can find $U \in C_{c}^{1}\left(\overline{\mathbb{R}_{+}^{d+1}}\right)$ depending linearly on $u$ and depending on $\beta$ such that $U(x, 0)=u(x)$ in $\mathbb{R}^{d}$

$$
\iint_{\mathbb{R}^{d} \times(0,+\infty)} \frac{\left|\nabla_{A} U(x, t)\right|^{p}}{t^{1-(1-s) p}} \mathrm{~d} x \mathrm{~d} t \leq C_{d, s, p}\left(|u|_{W_{A^{\prime \prime}}^{s, p}\left(\mathbb{R}^{d}\right)}^{p}+\beta^{\frac{s p}{2}}\|u\|_{L^{p}\left(\mathbb{R}^{d}\right)}^{p}\right)
$$

and

$$
\iint_{\mathbb{R}^{d} \times(0,+\infty)} \frac{|U(z, t)|^{p}}{t^{1-(1-s) p}} \mathrm{~d} x \mathrm{~d} t \leq \frac{C_{d, s, p}}{\beta^{\frac{(1-s) p}{2}}}\|u\|_{L^{p}\left(\mathbb{R}^{d}\right)}^{p} .
$$

Proof. Let $\varphi \in C_{c}^{\infty}\left(\mathbb{R}^{d}\right)$ and $\theta \in C^{\infty}(\mathbb{R})$ be functions such that

$$
\int_{\mathbb{R}^{d}} \varphi=1, \quad \varphi(x)=0 \text { for }|x|>1
$$

$$
\theta=1 \text { in }(-a / 2, a / 2), \quad \theta=0 \text { in } \mathbb{R} \backslash(-a, a), \quad \text { and } \quad\left|\theta^{\prime}\right| \leq C_{1} / a \text { in } \mathbb{R},
$$

with $a=\beta^{-1 / 2}$ for some positive constant $C_{1}$ independent of $a$, and set, for $t>0$

$$
\varphi_{t}(\cdot) \triangleq t^{-d} \varphi(\cdot / t) \quad \text { in } \mathbb{R}^{d}
$$

We define the function $U: \mathbb{R}_{+}^{d+1} \rightarrow \mathbb{C}$ by setting, for every $(x, t) \in \mathbb{R}_{+}^{d+1}$,

$$
U(x, t) \triangleq \theta(t) \int_{\mathbb{R}^{d}} \varphi_{t}(x-y) e^{i \mathcal{I}_{A}((x, t), y)} u(y) \mathrm{d} y
$$

where we have identified the point $(y, 0) \in \mathbb{R}^{d} \times\{0\} \subset \mathbb{R}^{d+1}$ with $y \in \mathbb{R}^{d}$.

Set, for $1 \leq j \leq d+1$,

$$
\left.H_{j}((x, t), y) \triangleq \frac{\mathrm{d}}{\mathrm{d} \tau} \mathcal{I}_{A}\left((x, t)+\tau e_{j}, y\right)\right|_{\tau=0}+A_{j}(x, t) \text { for }(x, t) \in \mathbb{R}_{+}^{d+1}, y \in \mathbb{R}^{d}
$$

For every $(x, t) \in \mathbb{R}_{+}^{d+1}$ and for $1 \leq j \leq d$, we have 


$$
\begin{aligned}
& \partial_{j} U(x, t)+i A_{j}(x, t) U(x, t) \\
&=\theta(t) \int_{\mathbb{R}^{d}}\left(\partial_{j} \varphi_{t}(x-y)+i \varphi_{t}(x-y) H_{j}((x, t), y)\right) e^{i \mathcal{I}_{A}((x, t), y)} u(y) \mathrm{d} y
\end{aligned}
$$

and

$$
\begin{aligned}
\partial_{d+1} U(x, t)+i A_{d+1}(x, t) U(x, t)=\int_{\mathbb{R}^{d}} & \left\{\left(\theta^{\prime}(t)-\theta(t)\left(\frac{d}{t}-i H_{d+1}((x, t), y)\right)\right) \varphi_{t}(x-y)\right. \\
& \left.\left.-\frac{\theta(t)}{t^{d+2}} \nabla \varphi\left(\frac{x-y}{t}\right) \cdot(x-y)\right)\right\} e^{i \mathcal{I}_{A}((x, t), y)} u(y) \mathrm{d} y .
\end{aligned}
$$

With the notations $\Phi_{t}^{j}(z) \triangleq \partial_{j} \varphi_{t}(z)$ if $1 \leq j \leq d$ and $\Phi_{t}^{d+1}(z)=-\frac{d}{t} \varphi_{t}(z)-$ $t^{-(d+2)} \nabla \varphi(z / t) \cdot z$ for $z \in \mathbb{R}^{d}$, we have, since $\int_{\mathbb{R}^{d}} \Phi_{t}^{j}=0$,

$$
\begin{aligned}
\int_{\mathbb{R}^{d}} \Phi_{t}^{j}(x-y) e^{i \mathcal{I}_{A}((x, t), y)} u(y) \mathrm{d} y & \\
= & \int_{\mathbb{R}^{d}} \Phi_{t}^{j}(x-y) e^{i \mathcal{I}_{A}((x, t), x)}\left(e^{i \mathcal{I}_{A^{\prime \prime}}(x, y)} u(y)-u(x)\right) \mathrm{d} y \\
& \quad+\int_{\mathbb{R}^{d}} \Phi_{t}^{j}(x-y)\left(e^{i \mathcal{I}_{A}((x, t), y)}-e^{i \mathcal{I}_{A}(x, y)+i \mathcal{I}_{A}((x, t), x)}\right) u(y) \mathrm{d} y .
\end{aligned}
$$

It follows that, for every $(x, t) \in \mathbb{R}_{+}^{d+1}$,

$$
\left|\nabla_{A} U(x, t)\right| \leq C_{2}\left(L_{1}(x, t)+L_{2}(x, t)+L_{3}(x, t)+L_{4}(x, t)\right),
$$

where the functions $L_{1}, L_{2}, L_{3}, L_{4}: \mathbb{R}_{+}^{d+1} \rightarrow \mathbb{R}$ are defined for each $(x, t) \in \mathbb{R}_{+}^{d+1}$ by

$$
\begin{aligned}
& L_{1}(x, t) \triangleq \frac{\mathbb{1}_{(0, a)}(t)}{t^{d+1}} \int_{B(x, t)}\left|e^{i \mathcal{I}_{A}(x, y)} u(y)-u(x)\right| \mathrm{d} y, \\
& L_{2}(x, t) \triangleq \frac{\mathbb{1}_{(0, a)}(t)}{t^{d+1}} \int_{B(x, t)}\left|e^{i\left(\mathcal{I}_{A}((x, t), y)+\mathcal{I}_{A}(y, x)+\mathcal{I}_{A}(x,(x, t))\right)}-1\right||u(y)| \mathrm{d} y, \\
& L_{3}(x, t) \triangleq \frac{\mathbb{1}_{(0, a)}(t)}{t^{d}} \int_{B(x, t)}|H((x, t), y)||u(y)| \mathrm{d} y, \\
& L_{4}(x, t) \triangleq \frac{\mathbb{1}_{(0, a)}(t)}{a t^{d}} \int_{B(x, t)}|u(y)| \mathrm{d} y .
\end{aligned}
$$


First, we have, by Hölder's inequality,

$$
\begin{aligned}
\iint_{\mathbb{R}_{+}^{d+1}} \frac{\left|L_{1}(x, t)\right|^{p}}{t^{1-(1-s) p}} \mathrm{~d} x \mathrm{~d} t & \leq C_{3} \iint_{\mathbb{R}_{+}^{d+1}} \frac{1}{t^{1+d+s p}}\left(\int_{B(x, t)}\left|e^{i \mathcal{I}_{A}(x, y)} u(y)-u(x)\right|^{p} \mathrm{~d} y\right) \mathrm{d} x \mathrm{~d} t \\
& \leq C_{4} \iint_{\mathbb{R}^{d} \times \mathbb{R}^{d}} \frac{\left|e^{i \mathcal{I}_{A^{\prime \prime}}(x, y)} u(y)-u(x)\right|^{p}}{|y-x|^{d+s p}} \mathrm{~d} x \mathrm{~d} y .
\end{aligned}
$$

Second, by Lemma 2.2 , if $x, y \in \mathbb{R}^{d}$ and $t \in(0,+\infty)$, we have

$$
\left|\mathcal{I}_{A}((x, t), y)+\mathcal{I}_{A}(y, x)+\mathcal{I}_{A}(x,(x, t))\right| \leq \frac{\beta t|y-x|}{2}
$$

and therefore, for $y \in B(x, t)$,

$$
\left|e^{i\left(\mathcal{I}_{A}((x, t), y)+\mathcal{I}_{A}(y, x)+\mathcal{I}_{A}(x,(x, t))\right)}-1\right| \leq C_{5} \beta^{1 / 2} t .
$$

It follows from Fubini's theorem that

$$
\begin{aligned}
\iint_{\mathbb{R}_{+}^{d+1}} \frac{\left|L_{2}(x, t)\right|^{p}}{t^{1-(1-s) p}} \mathrm{~d} x \mathrm{~d} t & \leq C_{6} \iint_{\mathbb{R}^{d} \times(0, a)} \frac{\beta^{p / 2}}{t^{d+1-(1-s) p}} \int_{B(x, t)}|u(y)|^{p} \mathrm{~d} y \mathrm{~d} x \mathrm{~d} t \\
& =C_{7} \int_{0}^{a} \frac{\beta^{\frac{p}{2}}}{t^{1-(1-s) p}} \int_{\mathbb{R}^{d}}|u(y)|^{p} \mathrm{~d} y \mathrm{~d} t=C_{8} \beta^{\frac{s p}{2}} \int_{\mathbb{R}^{d}}|u(y)|^{p} \mathrm{~d} y .
\end{aligned}
$$

Third, we have by Lemma 2.2 for $x, y \in \mathbb{R}^{d}$ and $t \in(0,+\infty)$,

$$
\begin{aligned}
& \mathcal{I}_{A}\left((x, t)+\tau e_{j}, y\right)-\mathcal{I}_{A}((x, t), y)=\mathcal{I}_{A}\left((x, t)+\tau e_{j},(x, t)\right) \\
& +\tau \int_{0}^{1} \int_{0}^{1-\sigma} d A\left((1-\sigma)(x, t)+\rho \tau e_{j}+\sigma(y, 0)\right)\left[e_{j},(y-x,-t)\right] \mathrm{d} \rho \mathrm{d} \sigma,
\end{aligned}
$$

and

$$
\mathcal{I}_{A}\left((x, t)+\tau e_{j},(x, t)\right)=-\tau \int_{0}^{1} A_{j}\left((x, t)+\sigma \tau e_{j}\right) \mathrm{d} \sigma
$$

It follows by (3.2) that

$$
H_{j}((x, t), y)=\int_{0}^{1} \int_{0}^{1-\sigma} d A((1-\sigma)(x, t)+\sigma(y, 0))\left[e_{j},(y-x,-t)\right] \mathrm{d} \sigma
$$


which yields, for $y \in B(x, t)$

$$
|H((x, t), y)| \leq C_{9} \beta t
$$

This implies, as in (3.6),

$$
\begin{aligned}
\iint_{\mathbb{R}^{d} \times(0,+\infty)} & \frac{\left|L_{3}(x, t)\right|^{p}}{t^{1-(1-s) p}} \mathrm{~d} x \mathrm{~d} t \\
& \leq C_{10} \iint_{\mathbb{R}^{d} \times(0, a)} \frac{\beta^{p} t^{p}}{t^{d+1-(1-s) p}} \int_{B(x, t)}|u(y)|^{p} \mathrm{~d} y \mathrm{~d} x \mathrm{~d} t=C_{11} \beta^{\frac{s p}{2}} \int_{\mathbb{R}^{d}}|u(y)|^{p} \mathrm{~d} y .
\end{aligned}
$$

Finally, we have

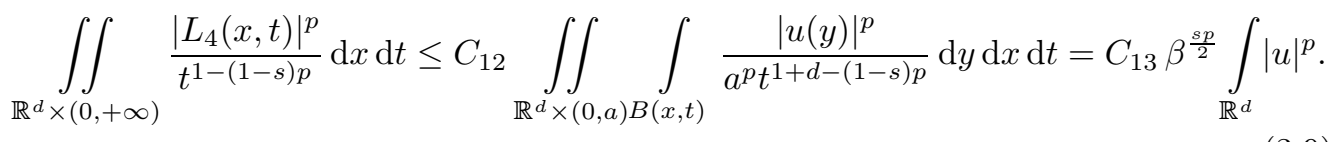

Combining (3.4), (3.5), (3.6), (3.8), and (3.9) yields

$$
\iint_{\mathbb{R}^{d} \times(0,+\infty)} \frac{\left|\nabla_{A} U(x, t)\right|^{p}}{t^{1-(1-s) p}} \mathrm{~d} x \mathrm{~d} t \leq C_{14}|u|_{W_{A^{\prime \prime}}^{s, p}\left(\mathbb{R}^{d}\right)}^{p}+C_{15} \beta^{\frac{s p}{2}}\|u\|_{L^{p}\left(\mathbb{R}^{d}\right)}^{p} .
$$

Similar to (3.9), we also have

$$
\iint_{\mathbb{R}^{d} \times(0,+\infty)} \frac{|U(x, t)|^{p}}{t^{1-(1-s) p}} \mathrm{~d} x \mathrm{~d} t \leq C_{16} \frac{\|u\|_{L^{p}\left(\mathbb{R}^{d}\right)}^{p}}{\beta^{\frac{(1-s) p}{2}}}
$$

The conclusion now follows from the estimates (3.10) and (3.11).

We also have a local version of Proposition 3.1.

Proposition 3.2. Let $d \geq 1,0<s<1$ and $1 \leq p<+\infty$. There exists a constant $C_{d, s, p}$ such that for every $u \in C^{1}\left(B_{2 R}, \mathbb{C}\right)$ and every $A \in C^{1}\left(\bar{B}_{2 R} \times[0, R], \bigwedge^{1} \mathbb{R}^{d+1}\right)$ such that $\|d A\|_{L^{\infty}\left(B_{2 R} \times[0, R]\right)}+\frac{1}{R^{2}} \leq \beta$, there exists $U \in C^{1}\left(\bar{B}_{R} \times[0, R], \mathbb{C}\right)$ such that $U(\cdot, 0)=u(\cdot)$ on $B_{R}$ and

$$
\begin{aligned}
& \iint_{B_{R} \times(0, R)} \frac{\left|\nabla_{A} U(x, t)\right|^{p}}{t^{1-(1-s) p}} \mathrm{~d} x \mathrm{~d} t \\
& \leq C_{d, s, p}\left(\int_{B_{2 R} \times B_{2 R}} \frac{\left|e^{i \mathcal{I}_{A^{\prime \prime}}(x, y)} u(y)-u(x)\right|^{p}}{|x-y|^{d+s p}} \mathrm{~d} x \mathrm{~d} y+\beta^{\frac{s p}{2}} \int_{B_{2 R}}|u|^{p}\right)
\end{aligned}
$$


and

$$
\iint_{B_{R} \times(0, R)} \frac{|U(z, t)|^{p}}{t^{1-(1-s) p}} \mathrm{~d} t \mathrm{~d} z \leq \frac{C_{d, s, p}}{\beta^{\frac{(1-s) p}{2}}} \int_{B_{2 R}}|u|^{p} .
$$

\section{Characterizations of trace spaces}

For $\gamma \in \mathbb{R}$, we define the weighted space

$$
W_{A, \gamma}^{1, p}\left(\mathbb{R}_{+}^{d+1}\right) \triangleq\left\{u \in W_{\mathrm{loc}}^{1,1}\left(\mathbb{R}_{+}^{d+1}, \mathbb{C}\right) ;\|U\|_{W_{A, \gamma}^{1, p}(\Omega)}<+\infty\right\}
$$

where

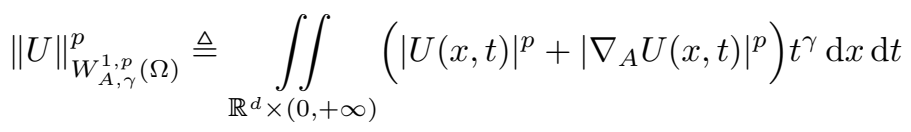

It is standard to check that the space $W_{A, \gamma}^{1, p}\left(\mathbb{R}_{+}^{d+1}\right)$ is complete. We also have the following density result:

Lemma 4.1. Let $1 \leq p<+\infty, \gamma \in \mathbb{R}$ and $A \in C\left(\mathbb{R}_{+}^{d+1}, \mathbb{R}^{d+1}\right)$. If $-1<\gamma<p-1$, then the space $C_{c}^{\infty}\left(\overline{\mathbb{R}_{+}^{d+1}}\right)$ is dense in $W_{A, \gamma}^{1, p}\left(\mathbb{R}_{+}^{d+1}\right)$.

Proof. The proof of the completeness is standard. For the density of smooth maps, we first observe that if $\chi \in C_{c}^{\infty}\left(\mathbb{R}^{d+1}\right), 0 \leq \chi \leq 1$, and $\chi=1$ on $B_{1}$ and if we define for each $\lambda>0$ the function $\chi_{\lambda}: \mathbb{R}^{d+1} \rightarrow \mathbb{R}$ for $x \in \mathbb{R}^{d}$ by $\chi_{\lambda}(x)=\chi(x / \lambda)$, then $\nabla_{A}\left(U-\chi_{\lambda} U\right)=-U \nabla \chi_{\lambda}+\left(1-\chi_{\lambda}\right) \nabla_{A} U$. It follows that

$$
\begin{aligned}
& \left\|U-\chi_{\lambda} U\right\|_{W_{A, \gamma}^{1, p}\left(\mathbb{R}^{d}\right)}^{p} \\
& \leq C_{1} \iint_{\mathbb{R}^{d} \times(0,+\infty)}\left(\left(1-\chi_{\lambda}(x, t)\right)^{p}\left(|U(x, t)|^{p}+\left|\nabla_{A} U(x, t)\right|^{p}\right)+\left|\nabla \chi_{\lambda}\right|^{p}|U(x, t)|^{p}\right) t^{\gamma} \mathrm{d} x \mathrm{~d} t \rightarrow 0,
\end{aligned}
$$

as $\lambda \rightarrow \infty$, since $\left|\nabla \chi_{\lambda}\right| \leq C_{2} / \lambda$. Functions in $W_{A, \gamma}^{1, p}\left(\mathbb{R}_{+}^{d+1}\right)$ with bounded support are thus dense in $W_{A, \gamma}^{1, p}\left(\mathbb{R}_{+}^{d+1}\right)$.

Since $-1<\gamma<p-1$, any function $W_{0, \gamma}^{1, p}\left(\mathbb{R}_{+}^{d+1}\right)$ can be extended by even reflection to $\mathbb{R}^{d+1}$ and can be approximated in $W_{0, \gamma}^{1, p}\left(\mathbb{R}^{d+1}\right)$ by smooth functions with bounded support [21, Lemma 2.4], [24, Lemma 8], [35, Corollary 2.1.5] (the condition ensures that the weight $(x, t) \in \mathbb{R}^{d+1} \mapsto|t|^{\gamma}$ satisfies Muckenhoupt's $A_{p}$ condition for $\left.p \in[1,+\infty)[7,23]\right)$. Since the function $A$ is locally bounded on $\mathbb{R}^{d+1}$, this implies that any bounded supported function in $W_{A, \gamma}^{1, p}\left(\mathbb{R}_{+}^{d+1}\right)$ can be approximated in $W_{0, \gamma}^{1, p}\left(\mathbb{R}_{+}^{d+1}\right)$ by smooth functions with bounded support and the conclusion follows by a diagonal argument. 
It is also standard to check that the space $W_{A^{\prime \prime}}^{s, p}\left(\mathbb{R}^{d}\right)$ is complete and thus is a Banach space. We also have the following density result:

Lemma 4.2. Let $0<s<1$ and $1 \leq p<+\infty$. If $A^{\prime \prime} \in C^{1}\left(\mathbb{R}^{d}, \mathbb{R}^{d}\right)$, then the space $C_{c}^{\infty}\left(\mathbb{R}^{d}\right)$ is dense in $W_{A^{\prime \prime}}^{s, p}\left(\mathbb{R}^{d}\right)$.

Proof. First we observe that if $u \in W_{A^{\prime \prime}}^{s, p}\left(\mathbb{R}^{d}\right)$ is arbitrary, $\chi \in C_{c}^{\infty}\left(\mathbb{R}^{d}\right)$ is chosen with $0 \leq \chi \leq 1$ and $\chi=1$ for $x \in B_{1}$, and $\chi_{\lambda}(x) \triangleq \chi(x / \lambda)$, then we have for each $x, y \in \mathbb{R}^{d}$ and $\lambda>0$,

$$
\begin{aligned}
& \left(1-\chi_{\lambda}(y)\right) e^{i \mathcal{I}_{A^{\prime \prime}}(x, y)} u(y)-\left(1-\chi_{\lambda}(x)\right) u(x) \\
& \quad=\left(1-\frac{\chi_{\lambda}(x)+\chi_{\lambda}(y)}{2}\right)\left(e^{i \mathcal{I}_{A^{\prime \prime}}(x, y)} u(y)-u(x)\right)+\frac{\chi_{\lambda}(x)-\chi_{\lambda}(y)}{2}\left(e^{i \mathcal{I}_{A^{\prime \prime}}(x, y)} u(y)+u(x)\right)
\end{aligned}
$$

and for every $y \in \mathbb{R}^{d}$ and $\lambda>0$,

$$
\int_{\mathbb{R}^{d}} \frac{\left|\chi_{\lambda}(x)-\chi_{\lambda}(y)\right|^{p}}{|y-x|^{d+s p}} \mathrm{~d} x \leq \frac{C_{1}}{\lambda^{s p}} .
$$

It follows that, for every $\lambda>0$,

$$
\begin{aligned}
& \left|\chi_{\lambda} u-u\right|_{W_{A^{\prime \prime}}^{s, p}\left(\mathbb{R}^{d}\right)}^{p} \\
& \quad \leq C_{2} \iint_{\mathbb{R}^{d} \times \mathbb{R}^{d}}\left(1-\frac{\chi_{\lambda}(x)+\chi_{\lambda}(y)}{2}\right)^{p} \frac{\left|e^{i \mathcal{I}_{A^{\prime \prime}}(x, y)} u(y)-u(x)\right|^{p}}{|y-x|^{d+s p}} \mathrm{~d} y \mathrm{~d} x+C_{3} \int_{\mathbb{R}^{d}} \frac{|u(y)|^{p}}{\lambda^{s p}} \mathrm{~d} y .
\end{aligned}
$$

By Lebesgue's dominated convergence theorem we deduce that

$$
\left|\chi_{\lambda} u-u\right|_{W_{A^{\prime \prime}}^{s, p}\left(\mathbb{R}^{d}\right)} \rightarrow 0 \quad \text { and } \quad\left\|\chi_{\lambda} u-u\right\|_{L^{p}\left(\mathbb{R}^{d}\right)} \rightarrow 0 \quad \text { as } \lambda \rightarrow+\infty
$$

Hence functions with compact support are dense in $W_{A^{\prime \prime}}^{s, p}\left(\mathbb{R}^{d}\right)$.

We conclude by observing that since $A^{\prime \prime}$ is locally bounded, any function in $W_{A^{\prime \prime}}^{s, p}\left(\mathbb{R}^{d}\right)$ with compact support is in $W_{0}^{s, p}\left(\mathbb{R}^{d}\right)$, such functions can be approximated in $W_{0}^{s, p}\left(\mathbb{R}^{d}\right)$ by functions with uniformly compact support; since the function $A^{\prime \prime}$ is locally bounded, the approximating sequence also converges in $W_{A^{\prime \prime}}^{s, p}\left(\mathbb{R}^{d}\right)$. The conclusion then follows by a diagonal argument.

As a consequence of Propositions 2.1 and 3.1, and Lemmas 4.1 and 4.2, we obtain

Theorem 4.3. Let $d \geq 1,0<s<1$ and $1 \leq p<+\infty$. If $A \in C^{1}\left(\overline{\mathbb{R}_{+}^{d+1}}, \mathbb{R}^{d+1}\right)$ and if $\|d A\|_{L^{\infty}\left(\mathbb{R}_{+}^{d+1}\right)} \leq \beta$, then there exists a trace mapping $\operatorname{Tr}: W_{A, 1-(1-s) p}^{1, p}\left(\mathbb{R}_{+}^{d+1}, \mathbb{C}\right) \rightarrow$ $W_{A^{\prime \prime}}^{s, p}\left(\mathbb{R}^{d}\right)$ such that for some positive constant $C_{d, s, p}$ depending only on $d, s$ and $p$, 
if $U \in C_{c}^{1}\left(\mathbb{R}_{+}^{d+1}, \mathbb{C}\right)$, then $\operatorname{Tr} U=U(\cdot, 0)$ and for every $U \in W_{A, 1-(1-s) p}^{1, p}\left(\mathbb{R}_{+}^{d+1}\right)$, if $u \triangleq \operatorname{Tr} U$

$$
|u|_{W_{A^{\prime \prime}}^{s, p}\left(\mathbb{R}^{d}\right)}^{p}+\beta^{\frac{s p}{2}}\|u\|_{L^{p}\left(\mathbb{R}^{d}\right)}^{p} \leq C_{d, s, p} \iint_{\mathbb{R}^{d} \times(0,+\infty)} \frac{\left|\nabla_{A} U(z, t)\right|^{p}+\beta^{\frac{p}{2}}|U(z, t)|^{p}}{t^{1-(1-s) p}} \mathrm{~d} z \mathrm{~d} t
$$

and there exists a linear continuous mapping Ext $: W_{A^{\prime \prime}}^{s, p}\left(\mathbb{R}^{d}, \mathbb{C}\right) \rightarrow W_{A, 1-(1-s) p}^{1, p}\left(\mathbb{R}_{+}^{d+1}\right)$ such that $\operatorname{Tr} \circ \operatorname{Ext}: W_{A^{\prime \prime}}^{s, p}\left(\mathbb{R}^{d}\right) \rightarrow W_{A^{\prime \prime}}^{s, p}\left(\mathbb{R}^{d}\right)$ is the identity and such that for some positive constant $C_{d, s, p}$ depending only on $d, s$ and $p$, we have for each $u \in W_{A^{\prime \prime}}^{s, p}\left(\mathbb{R}^{d}\right)$, if $U \triangleq$ Ext $u$,

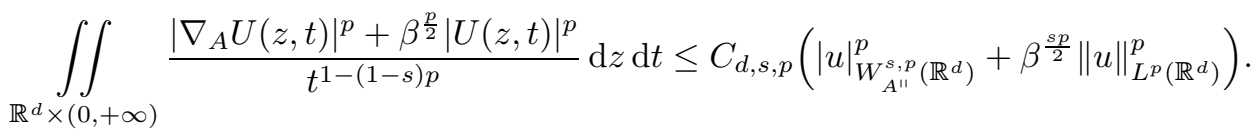

\section{Constant magnetic field on the half-space}

We begin with an improvement of Proposition 2.1 in the case where the magnetic field $d A$ is constant.

Proposition 5.1. Let $d \geq 1,0<s<1$, and $1 \leq p<+\infty$. There exists a constant $C_{d, s, p}>0$ such that if $A \in C^{1}\left(\mathbb{R}_{+}^{d+1}, \mathbb{R}^{d+1}\right)$ with constant $d A, U \in C_{c}^{1}\left(\overline{\mathbb{R}_{+}^{d+1}}\right)$ and $u=U(\cdot, 0)$, then

$$
|u|_{W_{A^{\prime \prime}}^{s, p}\left(\mathbb{R}^{d}\right)}^{p}+\left\|d A^{\prime \prime}\right\|{ }^{\frac{s p}{2}} \int_{\mathbb{R}^{d}}|u|^{p} \leq C_{d, s, p} \iint_{\mathbb{R}^{d} \times(0,+\infty)} \frac{\left|\nabla_{A} U(z, t)\right|^{p}}{t^{1-(1-s) p}} \mathrm{~d} z \mathrm{~d} t .
$$

The first ingredient of the proof Proposition 5.1 is the following lemma:

Lemma 5.2. Let $d \geq 1,0<s<1$, and $1 \leq p<+\infty$. For every $\lambda>0$, there exists a positive constant $C_{d, s, p, \lambda}$ such that, for every $A \in C^{1}\left(\overline{\mathbb{R}_{+}^{d+1}}, \bigwedge^{1} \mathbb{R}^{d+1}\right)$ with constant $d A$, we have, for $U \in C_{c}^{1}\left(\overline{\mathbb{R}_{+}^{d+1}}, \mathbb{C}\right)$,

$$
\begin{aligned}
\iint_{\mathbb{R}^{d} \times \mathbb{R}^{d}} \frac{\left|e^{i\left(\mathcal{I}_{A^{\prime \prime}}(x, y)-\lambda|y-x| d A\left[y-x, e_{d+1}\right]\right)} U(y, 0)-U(x, 0)\right|^{p}}{|y-x|^{d+s p}} \mathrm{~d} x \mathrm{~d} y & \leq C_{d, s, p, \lambda} \iint_{\mathbb{R}^{d} \times(0,+\infty)} \frac{\left|\nabla_{A} U(z, t)\right|^{p}}{t^{1-(1-s) p} \mathrm{~d} z \mathrm{~d} t .}
\end{aligned}
$$

Proof. For $x, y \in \mathbb{R}^{d}$, we identify $x=(x, 0), y=(y, 0)$, and we set $Z=\left(\frac{x+y}{2}, 2 \lambda|y-x|\right)$. Since $d A$ is constant, by Lemma 2.2 , we have 


$$
\mathcal{I}_{A}(x, y)+\mathcal{I}_{A}(y, Z)+\mathcal{I}_{A}(Z, x)=\frac{1}{2} d A\left[(y-x, 0),\left(\frac{y-x}{2}, 2 \lambda|x-y|\right)\right]=\lambda \nu_{A}(y-x),
$$

where the function $\nu_{A}: \mathbb{R}^{d} \rightarrow \mathbb{R}$ is defined for each $z \in \mathbb{R}^{d}$ by $\nu_{A}(z)=|z| \mathrm{d} A\left[z, e_{d+1}\right]$. This implies by the triangle inequality

$$
\left|e^{i\left(\mathcal{I}_{A}(x, y)-\lambda \nu_{A}(y-x)\right)} U(y)-U(x)\right| \leq\left|e^{i \mathcal{I}_{A}(x, Z)} U(Z)-U(x)\right|+\left|e^{i \mathcal{I}_{A}(y, Z)} U(Z)-U(y)\right| .
$$

Using (2.6), we deduce from (5.1) that

$$
\begin{aligned}
&\left|e^{i\left(\mathcal{I}_{A}(x, y)-\lambda \nu_{A}(y-x)\right)} U(y)-U(x)\right| \\
& \leq \sqrt{1+4 \lambda^{2}}|y-x|\left(\int_{0}^{1}\left|\nabla_{A} U\left(\left(1-\frac{t}{2}\right) x+\frac{t}{2} y, 2 \lambda t|y-x|\right)\right| \mathrm{d} t\right. \\
&\left.+\int_{0}^{1}\left|\nabla_{A} U\left(\left(1-\frac{t}{2}\right) y+\frac{t}{2} x, 2 \lambda t|y-x|\right)\right| \mathrm{d} t\right) .
\end{aligned}
$$

We then have, by Minkowski's inequality,

$$
\begin{aligned}
& \iint_{\mathbb{R}^{d} \times \mathbb{R}^{d}} \frac{\left|e^{i\left(\mathcal{I}_{A}(x, y)-\lambda \nu_{A}(y-x)\right)} U(y)-U(x)\right|^{p}}{|y-x|^{d+s p}} \mathrm{~d} x \mathrm{~d} y \\
& \leq C_{1}(1+\lambda)^{p}\left\{\int_{0}^{1}\left(\iint_{\mathbb{R}^{d} \times \mathbb{R}^{d}} \frac{\left|\nabla_{A} U\left(\left(1-\frac{t}{2}\right) x+\frac{t}{2} y, 2 \lambda t|y-x|\right)\right|^{p}}{|y-x|^{d-(1-s) p}} \mathrm{~d} y \mathrm{~d} x\right)^{1 / p}\right. \\
& \left.+\left(\iint_{\mathbb{R}^{d} \times \mathbb{R}^{d}} \frac{\left|\nabla_{A} U\left(\left(1-\frac{t}{2}\right) y+\frac{t}{2} x, 2 \lambda t|y-x|\right)\right|^{p}}{|y-x|^{d-(1-s) p}} \mathrm{~d} y \mathrm{~d} x\right)^{\frac{1}{p}} \mathrm{~d} t\right\}^{p} .
\end{aligned}
$$

Performing the change of variable $z=\left(1-\frac{t}{2}\right) x+\frac{t}{2} y$ and $v=2 \lambda t(x-y)$, we obtain

$$
\begin{aligned}
\iint_{\mathbb{R}^{d} \times \mathbb{R}^{d}} \frac{\left|\nabla_{A} U\left(\left(1-\frac{t}{2}\right) x+\frac{t}{2} y, 2 \lambda t|y-x|\right)\right|^{p}}{|y-x|^{d-(1-s) p}} \mathrm{~d} y \mathrm{~d} x & \\
& =\frac{1}{(2 \lambda t)^{(1-s) p}} \iint_{\mathbb{R}^{d} \times \mathbb{R}^{d}} \frac{\left|\nabla_{A} U(z,|v|)\right|^{p}}{|v|^{d-(1-s) p} \mathrm{~d} z \mathrm{~d} v .}
\end{aligned}
$$

This yields by using spherical coordinates, for every $t \in(0,1)$, 
$\iint_{\mathbb{R}^{d} \times \mathbb{R}^{d}} \frac{\left|\nabla_{A} U\left(\left(1-\frac{t}{2}\right) x+\frac{t}{2} y, 2 \lambda t|y-x|\right)\right|^{p}}{|y-x|^{d-(1-s) p}} \mathrm{~d} x \mathrm{~d} y \leq \frac{C_{2}}{(\lambda t)^{(1-s) p}} \iint_{\mathbb{R}^{d} \times(0,+\infty)} \frac{\left|\nabla_{A} U(z, r)\right|^{p}}{r^{1-(1-s) p}} \mathrm{~d} z \mathrm{~d} r$.

Similarly, we have

$$
\iint_{\mathbb{R}^{d} \times \mathbb{R}^{d}} \frac{\left|\nabla_{A} U\left(\left(1-\frac{t}{2}\right) y+\frac{t}{2} x, 2 \lambda t|y-x|\right)\right|^{p}}{|y-x|^{d-(1-s) p}} \mathrm{~d} x \mathrm{~d} y \leq \frac{C_{2}}{(\lambda t)^{(1-s) p}} \iint_{\mathbb{R}^{d} \times(0,+\infty)} \frac{\left|\nabla_{A} U(z, r)\right|^{p}}{r^{1-(1-s) p}} \mathrm{~d} z \mathrm{~d} r .
$$

Combining (5.2) with (5.3) and (5.4) yields

$$
\begin{aligned}
& \iint_{\mathbb{R}^{d} \times \mathbb{R}^{d}} \frac{\left|e^{i\left(\mathcal{I}_{A^{\prime \prime}}(x, y)-\lambda \nu_{A}(y-x)\right)} U(y, 0)-U(x, 0)\right|^{p}}{|y-x|^{d+s p}} \mathrm{~d} x \mathrm{~d} y \\
& \leq \frac{C_{3}(1+\lambda)^{p}}{\lambda^{(1-s) p}}\left(\int_{0}^{1} \frac{1}{t^{1-s}} \mathrm{~d} t\right)^{p} \underset{\mathbb{R}^{d} \times(0,+\infty)}{\iint_{r^{1-(1-s) p}}} \frac{\left|\nabla_{A} U(z, r)\right|^{p}}{r^{1-(1-\infty)}} \mathrm{d} r
\end{aligned}
$$

and the conclusion follows.

The second tool is the following fractional magnetic Poincaré inequality.

Lemma 5.3. Let $d \geq 1,0<s<1$ and $1 \leq p<+\infty$ and $A^{\prime \prime} \in C^{1}\left(\mathbb{R}^{d}, \bigwedge^{1} \mathbb{R}^{d}\right)$ with constant $d A^{\prime \prime}$. There exists a constant $C_{d, s, p}>0$ such that, for $u \in C_{c}^{1}\left(\mathbb{R}^{d}\right)$,

$$
\int_{\mathbb{R}^{d}}|u|^{p} \leq \frac{C_{d, s, p}}{\left\|d A^{\prime \prime}\right\|^{\frac{s p}{2}}} \iint_{\mathbb{R}^{d} \times \mathbb{R}^{d}} \frac{\left|e^{i \mathcal{I}_{A^{\prime \prime}}(x, y)} u(y)-u(x)\right|^{p}}{|y-x|^{d+s p}} \mathrm{~d} x \mathrm{~d} y .
$$

The counterpart of Lemma 5.3 in $W_{A}^{1,2}\left(\mathbb{R}^{d}\right)$ is known [4, Theorem 2.9], [11, Proposition 2.2] and related to the positiveness of the first eigenvalue of the magnetic Laplacian $-\Delta_{A}$, which corresponds to the first Landau level.

Proof of Lemma 5.3. Let $J: \mathbb{R}^{d} \rightarrow \mathbb{R}^{d}$ be a linear isometry. We observe that by setting $z=x+h$ and $k=J h$, we have

$$
\begin{aligned}
\iint_{\mathbb{R}^{d} \times \mathbb{R}^{d}} \frac{\left|e^{i \mathcal{I}_{A}(x+h, x+h+J h)} u(x+h+J h)-u(x+h)\right|^{p}}{|h|^{d+s p}} \mathrm{~d} x \mathrm{~d} h \\
=\iint_{\mathbb{R}^{d} \times \mathbb{R}^{d}} \frac{\left|e^{i \mathcal{I}_{A}(z, z+k)} u(z+k)-u(z)\right|^{p}}{|k|^{d+s p}} \mathrm{~d} z \mathrm{~d} k .
\end{aligned}
$$

Similarly, by setting $z=x+h+J h$ and $k=-h$, we have 


$$
\begin{array}{rl}
\iint_{\mathbb{R}^{d} \times \mathbb{R}^{d}} \frac{\mid e^{i \mathcal{I}_{A}(x+h+J h, x+J h)} u(x+J h)}{}-\left.u(x+h+J h)\right|^{p} & \mathrm{~d} x \mathrm{~d} h \\
|h|^{d+s p} & =\iint_{\mathbb{R}^{d} \times \mathbb{R}^{d}} \frac{\left|e^{i \mathcal{I}_{A}(z, z+k)} u(z+k)-u(z)\right|^{p}}{|k|^{d+s p}} \mathrm{~d} z \mathrm{~d} k .
\end{array}
$$

Finally, we have by setting $z=x+J h$ and $k=-J h$,

$$
\begin{aligned}
\iint_{\mathbb{R}^{d} \times \mathbb{R}^{d}} \frac{\left|e^{i \mathcal{I}_{A}(x+J h, x)} u(x)-u(x+J h)\right|^{p}}{|h|^{d+s p}} \mathrm{~d} x \mathrm{~d} h & \\
& =\iiint_{\mathbb{R}^{d} \times \mathbb{R}^{d}} \frac{\left|e^{i \mathcal{I}_{A}(z, z+k)} u(z+k)-u(z)\right|^{p}}{|k|^{d+s p}} \mathrm{~d} z \mathrm{~d} k .
\end{aligned}
$$

We compute now, since $d A$ is constant, by Stokes' formula

$\mathcal{I}_{A}(x, x+h)+\mathcal{I}_{A}(x+h, x+h+J h)+\mathcal{I}_{A}(x+h+J h, x+J h)+\mathcal{I}_{A}(x+J h, x)=d A[h, J h]$, so that, by the triangle inequality,

$$
\begin{aligned}
&\left|e^{i d A[h, J h]}-1\right||u(x)| \\
& \leq\left|e^{i \mathcal{I}_{A}(x, x+h)} u(x+h)-u(x)\right|+\left|e^{i \mathcal{I}_{A}(x+h, x+h+J h)} u(x+h+J h)-u(x+h)\right| \\
&+\left|e^{i \mathcal{I}_{A}(x+h+J h, x+J h)} u(x+J h)-u(x+h+J h)\right| \\
&+\left|e^{i \mathcal{I}_{A}(x+J h, x)} u(x)-u(x+J h)\right| .
\end{aligned}
$$

Therefore, we have by Minkowski's inequality, in view of (5.6), (5.7) and (5.8),

$$
\iint_{\mathbb{R}^{d} \times \mathbb{R}^{d}} \frac{\left|e^{i d A[h, J h]}-1\right|^{p}|u(x)|^{p}}{|h|^{d+s p}} \mathrm{~d} x \mathrm{~d} h \leq 4^{p} \iint_{\mathbb{R}^{d} \times \mathbb{R}^{d}} \frac{\left|e^{i \mathcal{I}_{A}(x, x+h)} u(x+h)-u(x)\right|^{p}}{|h|^{d+s p}} \mathrm{~d} x \mathrm{~d} h .
$$

Integrating with respect to $J$ over the group $S O_{d}$ of rotations of $\mathbb{R}^{d}$, we obtain

$$
\left\|d A^{\prime \prime}\right\|^{\frac{s p}{2}} \int_{\mathbb{R}^{d}}|u(x)|^{p} \mathrm{~d} x \leq C_{1} \iint_{\mathbb{R}^{d} \times \mathbb{R}^{d}} \frac{\left|e^{i \mathcal{I}_{A}(x, x+h)} u(x+h)-u(x)\right|^{p}}{|h|^{d+s p}} \mathrm{~d} x \mathrm{~d} h .
$$

The proof is complete.

We are ready to give 
Proof of Proposition 5.1. Applying Lemma 5.2 with $\lambda=1$ and $\lambda=2$, and using the triangle inequality, we obtain

$$
\iint_{\mathbb{R}^{d} \times \mathbb{R}^{d}} \frac{\left|e^{i|y-x| d A\left[y-x, e_{d+1}\right]}-1\right|^{p}|U(y, 0)|^{p}}{|y-x|^{d+s p}} \mathrm{~d} x \mathrm{~d} y \leq C_{2} \iint_{\mathbb{R}_{+}^{d+1}} \frac{\left|\nabla_{A} U(z, t)\right|^{p}}{t^{1-(1-s) p}} \mathrm{~d} z \mathrm{~d} t .
$$

Since

$$
\begin{gathered}
\left|e^{i \mathcal{I}_{A^{\prime \prime}}(x, y)} U(y, 0)-U(x, 0)\right|^{p} \leq 2^{p-1}\left|e^{i\left(\mathcal{I}_{A^{\prime \prime}}(x, y)-|y-x| d A\left[y-x, e_{d+1}\right]\right)} U(y, 0)-U(x, 0)\right|^{p} \\
+2^{p-1}\left|e^{-i|y-x| d A\left[y-x, e_{d+1}\right]}-1\right|^{p}|U(y, 0)|^{p}
\end{gathered}
$$

it follows from (5.9) and Lemma 5.2 with $\lambda=1$ that

$$
\iint_{\mathbb{R}^{d} \times \mathbb{R}^{d}} \frac{\left|e^{i \mathcal{I}_{A^{\prime \prime}}(x, y)} U(y, 0)-U(x, 0)\right|^{p}}{|y-x|^{d+s p}} \mathrm{~d} x \mathrm{~d} y \leq C_{3} \iint_{\mathbb{R}_{+}^{d+1}} \frac{\left|\nabla_{A} U(z, t)\right|^{p}}{t^{1-(1-s) p}} \mathrm{~d} z \mathrm{~d} t .
$$

From Lemma 5.3, we have

$$
\left\|d A^{\prime \prime}\right\|^{\frac{s p}{2}} \int_{\mathbb{R}^{d}}|U(\cdot, 0)| \leq C_{4} \iint_{\mathbb{R}_{+}^{d+1}} \frac{\left|\nabla_{A} U(z, t)\right|^{p}}{t^{1-(1-s) p}} \mathrm{~d} z \mathrm{~d} t
$$

Combining (5.10) and (5.11) yields the conclusion.

Using Proposition 5.1 and Theorem 4.3, we obtain the following result which implies Theorem 1.3 in the introduction.

Theorem 5.4. Let $d \geq 1,0<s<1$, and $1 \leq p<+\infty$. Assume that $A \in C^{1}\left(\overline{\mathbb{R}_{+}^{d+1}}, \mathbb{R}^{d+1}\right)$ and $d A$ is constant. Then, with $u \triangleq \operatorname{Tr} U$,

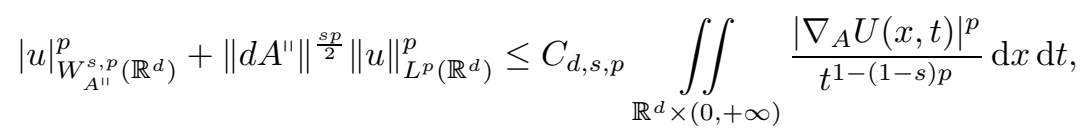

and with $U \triangleq \operatorname{Ext} u$,

$$
\iint_{\mathbb{R}^{d} \times(0,+\infty)} \frac{\left|\nabla_{A} U(z, t)\right|^{p}+\|d A\|^{\frac{p}{2}}|U(z, t)|^{p}}{t^{1-(1-s) p}} \mathrm{~d} z \mathrm{~d} t \leq C_{d, s, p}|u|_{W_{A^{\prime \prime}}^{s, p}\left(\mathbb{R}^{d}\right)}^{p}
$$

for some positive constant $C_{d, s, p}$ depending only on $d, s$ and $p$. 


\section{Trace and extension on domains}

In this section, we consider the trace problem on a domain $\Omega \subset \mathbb{R}^{d+1}$ of class $C^{1}$ with estimates depending only on the magnetic field $d A$. We first develop the tools to work with a magnetic derivative on the boundary $\partial \Omega$ via local charts. Let $W \subset \mathbb{R}^{d+1}$ be an open set, $\psi \in C^{1}\left(W, \mathbb{R}^{d+1}\right)$, and $A: \psi(W) \rightarrow \mathbb{R}^{d+1}$. The pull-back $\psi^{*} A$ of $A$ by $\psi$ is defined for each $x \in W$ by

$$
\left(\psi^{*} A\right)(x) \triangleq D \psi(x)^{*} A(\psi(x)),
$$

where $D \psi(x)^{*}$ is the adjoint of $D \psi(x)$. We first recall the following elementary result whose proof follows from the chain rule (see, e.g., [38, Proposition 6.1.11]) and the definition of the pull-back (6.1).

Lemma 6.1. Let $d \geq 1, V \subset \mathbb{R}^{d+1}$ be open, bounded, and let $\psi \in C^{1}\left(V, \mathbb{R}^{d+1}\right)$ be a diffeomorphism on its image up to the boundary. Then $U \in W_{A}^{1, p}(\psi(V))$ if and only if $U \circ \psi \in W_{\psi^{*} A}^{1, p}(V)$. Moreover, for almost every $x \in V$,

$$
\nabla_{\psi^{*} A}(U \circ \psi)(x)=D \psi(x)^{*}\left[\left(\nabla_{A} U\right)(\psi(x))\right]
$$

Consequently,

$$
\frac{\left|\nabla_{A} U(\psi(x))\right|}{\left\|D \psi^{-1}\right\|_{L^{\infty}}} \leq\left|\nabla_{\psi^{*} A}(U \circ \psi)(x)\right| \leq\|D \psi\|_{L^{\infty}}\left|\nabla_{A} U(\psi(x))\right|
$$

We next derive a similar result on the boundary when $\psi: W \mapsto \partial \Omega$ for the fractional magnetic Gagliardo-Sobolev seminorm. Although the potential $\mathcal{I}_{A}$ defined in (2.4) does not make sense in general for each $x, y \in \partial \Omega$ if $\Omega$ is not convex for $A^{\prime \prime} \in C\left(\bigwedge^{1} T^{*} \partial \Omega\right)$, when $\partial \Omega$ is compact smooth manifold, then $\partial \Omega$ has a positive injectivity radius inj $_{\partial \Omega}$ and if $x, y \in \partial \Omega$ and $d_{\partial \Omega}(y, x)<\operatorname{inj}_{\partial \Omega}$, then there exists a unique minimizing geodesic $\gamma:[0,1] \rightarrow \partial \Omega$ such that $\gamma(0)=x$ and $\gamma(1)=y$. We then define for such $x$ and $y$ the quantity

$$
\mathcal{I}_{A^{\prime \prime}}^{\partial \Omega}(x, y) \triangleq \int_{0}^{1} A^{\prime \prime}(\gamma(t)) \cdot \gamma^{\prime}(t) \mathrm{d} t
$$

We have

Lemma 6.2. Let $d \geq 1, W \subset \mathbb{R}^{d}$ be open, bounded, and let $\psi: W \rightarrow \mathbb{R}^{d+1}$ be a diffeomorphism up to the boundary to its image as a subset of the manifold $\partial \Omega$. If $W$ and $\psi(W)$ are geodesically convex, then for every $0<s<1$ and $1 \leq p<+\infty$, there exists a positive constant $C$ such that for every $A^{\prime \prime} \in C^{1}\left(\bigwedge^{1} T^{*} \partial \Omega\right)$ and every measurable function $u: \psi(W) \rightarrow \mathbb{C}$, 


$$
\begin{gathered}
C^{-1} \iint_{\psi(W) \times \psi(W)} \frac{\left|e^{i \mathcal{I}_{A^{\prime \prime}}^{\partial \Omega}(x, y)} u(y)-u(x)\right|^{p}}{d_{\partial \Omega}(y, x)^{d+s p}} \mathrm{~d} y \mathrm{~d} x \\
\leq \iint_{W \times W} \frac{\left|e^{i \mathcal{I}_{\psi^{*} A^{\prime \prime}}(x, y)} u(\psi(y))-u(\psi(x))\right|^{p}}{|y-x|^{d+s p}} \mathrm{~d} y \mathrm{~d} x \\
\quad+\min \left(\left\|d A^{\prime \prime}\right\|_{L^{\infty}}^{p},\left\|d A^{\prime \prime}\right\|_{L^{\infty}}^{\frac{s p}{3}}\right) \int_{W}|u(\psi(x))|^{p} \mathrm{~d} x
\end{gathered}
$$

and

$$
\begin{gathered}
\iint_{W \times W} \frac{\left|e^{i \mathcal{I}_{\psi^{*} A^{\prime \prime}}(x, y)} u(\psi(y))-u(\psi(x))\right|^{p}}{|y-x|^{d+s p}} \mathrm{~d} y \mathrm{~d} x \\
\leq C\left(\iint_{\psi(W) \times \psi(W)} \frac{\left|e^{i \mathcal{I}_{A^{\prime \prime}}^{\partial \Omega}(x, y)} u(y)-u(x)\right|^{p}}{d_{\partial \Omega}(y, x)^{d+s p}} \mathrm{~d} y \mathrm{~d} x\right. \\
\left.\quad+\min \left\{\left\|d A^{\prime \prime}\right\|_{L^{\infty}}^{p},\left\|d A^{\prime \prime}\right\|_{L^{\infty}}^{\frac{s p}{3}}\right\} \int_{\psi(W)}|u(x)|^{p} \mathrm{~d} x\right) .
\end{gathered}
$$

In the proof of Lemma 6.2, we use the following result which relates the potential $\mathcal{I}_{A}^{\partial \Omega}$ to the potential $\mathcal{I}_{\psi^{*} A}$ via local charts.

Lemma 6.3. Let $d \geq 1$, let $W \subset \mathbb{R}^{d}$ be open and bounded, and let $\psi: W \rightarrow \partial \Omega$ be a diffeomorphism up to the boundary to its image. If $W$ and $\psi(W)$ are geodesically convex, then there exists a positive constant $C$ such that for every $x, y \in W$, we have

$$
\left|\mathcal{I}_{\psi^{*} A^{\prime \prime}}(x, y)-\mathcal{I}_{A^{\prime \prime}}^{\partial \Omega}(\psi(x), \psi(y))\right| \leq C\left\|d A^{\prime \prime}\right\|_{L^{\infty}(\psi(W))}|y-x|^{3} .
$$

Proof. For every $x, y \in W$, there exists a unique minimizing geodesic $\gamma:[0,1] \rightarrow \psi(W)$ such that $\gamma(0)=\psi(x)$ and $\gamma(1)=\psi(y)$. Since $\gamma$ is a geodesic, the function $\tilde{\gamma}=\psi^{-1} \circ \gamma$ satisfies the equation

$$
\tilde{\gamma}^{\prime \prime}(t)=\Gamma(\tilde{\gamma}(t))\left[\tilde{\gamma}^{\prime}(t), \tilde{\gamma}^{\prime}(t)\right]
$$

where for every $z \in \psi(W), \Gamma(z)$ is a symmetric bilinear mapping (see, e.g., [10, Chapter $3]$ ). There exists thus a constant $C_{1}$ such that for every $t \in[0,1]$,

$$
\left|\tilde{\gamma}^{\prime \prime}(t)\right| \leq C_{1}|y-x|^{2}
$$

Since $\tilde{\gamma}(0)=x$ and $\tilde{\gamma}(1)=y$, we deduce that for every $t \in[0,1]$ we have

$$
|(1-t) x+t y-\tilde{\gamma}(t)| \leq C_{2} t(1-t)|y-x|^{2} .
$$


We have then by the Stokes theorem

$$
\mathcal{I}_{\psi^{*} A^{\prime \prime}}(y, x)-\mathcal{I}_{A^{\prime \prime}}^{\partial \Omega}(\psi(y), \psi(x))=\int_{[0,1]^{2}} d H^{*} A
$$

with the homotopy $H:[0,1]^{2} \rightarrow \mathbb{R}^{d}$ defined for each $(t, s) \in[0,1]^{2}$ by

$$
H(t, s) \triangleq(1-s)((1-t) x+t y)+s \tilde{\gamma}(t)
$$

and therefore,

$$
\left|\mathcal{I}_{\psi^{*} A^{\prime \prime}}(y, x)-\mathcal{I}_{A^{\prime \prime}}^{\partial \Omega}(\psi(y), \psi(x))\right| \leq C_{3}\left\|d A^{\prime \prime}\right\|_{L^{\infty}(\psi(W))}|y-x|^{3} .
$$

We are ready to give the

Proof of Lemma 6.2. By the change of variable formula, we have

$$
\begin{gathered}
\iint_{\psi(W) \times \psi(W)} \frac{\left|e^{i \mathcal{I}_{A^{\prime \prime}}^{\partial \Omega}(x, y)} u(y)-u(x)\right|^{p}}{d_{\partial \Omega}(y, x)^{d+s p}} \mathrm{~d} y \mathrm{~d} x \\
=\iint_{W \times W} \frac{\left|e^{i \mathcal{I}_{A^{\prime \prime}}^{\partial \Omega}(\psi(x), \psi(y))} u(\psi(y))-u(\psi(x))\right|^{p}}{d_{\partial \Omega}(\psi(y), \psi(x))^{d+s p}} \operatorname{Jac} \psi(y) \operatorname{Jac} \psi(x) \mathrm{d} y \mathrm{~d} x .
\end{gathered}
$$

We thus obtain

$$
\begin{gathered}
\iint_{\psi(W) \times \psi(W)} \frac{\left|e^{i \mathcal{I}_{A^{\prime \prime}}^{\partial \Omega}(x, y)} u(y)-u(x)\right|^{p}}{d_{\partial \Omega}(y, x)^{d+s p}} \mathrm{~d} y \mathrm{~d} x \\
\leq C_{1}\left(\iint_{W \times W} \frac{\left|e^{i \mathcal{I}_{\psi^{*} A^{\prime \prime}}(x, y)} u(\psi(y))-u(\psi(x))\right|^{p}}{|y-x|^{d+s p}} \mathrm{~d} y \mathrm{~d} x\right. \\
\left.\quad+\iint_{W \times W} \frac{\left|e^{i \mathcal{I}_{A^{\prime \prime}}^{\partial \Omega}(\psi(x), \psi(y))}-e^{i \mathcal{I}_{\psi^{*} A^{\prime \prime}}(x, y)}\right|^{p}|u(\psi(y))|^{p}}{|y-x|^{d+s p}} \mathrm{~d} y \mathrm{~d} x\right) .
\end{gathered}
$$

Since, by Lemma 6.3, for every $x, y \in W$,

$$
\frac{\left|e^{i \mathcal{I}_{A^{\prime \prime}}^{\partial \Omega}(\psi(x), \psi(y))}-e^{i \mathcal{I}_{\psi^{*} A^{\prime \prime}}(x, y)}\right|^{p}}{|y-x|^{d+s p}} \leq \frac{C_{2} \min \left\{\left\|d A^{\prime \prime}\right\|_{L^{\infty}(\psi(W))}^{p}|y-x|^{3 p}, 1\right\}}{|y-x|^{d+s p}},
$$

the first estimate then follows from the facts

$$
\int_{\mathbb{R}^{d}} \frac{\min \left\{\left\|d A^{\prime \prime}\right\|_{L^{\infty}(\psi(W))}^{p}|z|^{3 p}, 1\right\}}{|z|^{d+s p}} \mathrm{~d} z \leq C_{3}\left\|d A^{\prime \prime}\right\|_{L^{\infty}(\psi(W))}^{\frac{s p}{3}}
$$


and

$$
\int_{B(0, \operatorname{diam}(W))} \frac{\left\|d A^{\prime \prime}\right\|_{L^{\infty}(\psi(W))}^{p}|z|^{3 p}}{|z|^{d+s p}} \mathrm{~d} z \leq C_{4}\left\|d A^{\prime \prime}\right\|_{L^{\infty}(\psi(W))}^{p} .
$$

The proof of the second estimate follows similarly.

We have the following estimate on the traces of magnetic Sobolev spaces.

Proposition 6.4. Let $d \geq 1$, let $1 \leq p<+\infty$ and let $\Omega \subset \mathbb{R}^{d+1}$ be a bounded domain of class $C^{1}$. There exists a constant $C_{\Omega, p}>0$ such that if $A \in C^{1}\left(\bar{\Omega}, \bigwedge^{1} \mathbb{R}^{d}\right)$ and $\|d A\|_{L^{\infty}(\Omega)} \leq \beta$ and $U \in W_{A}^{1, p}(\Omega)$, then $u \triangleq \operatorname{Tr} U$ satisfies the estimate

$$
\iint_{\substack{(x, y) \in \partial \Omega \times \partial \Omega \\ d_{\partial \Omega}(y, x)<\operatorname{inj}_{\partial \Omega}}} \frac{\left|e^{i \mathcal{I}_{A^{\prime \prime}}^{\partial \Omega}(x, y)} u(y)-u(x)\right|^{p}}{|y-x|^{d+p-1}} \mathrm{~d} x \mathrm{~d} y \leq C_{\Omega, p} \int_{\Omega}\left|\nabla_{A} U\right|^{p}+\left(1+\beta^{\frac{p}{2}}\right)|U|^{p} .
$$

Here for $z \in \partial \Omega, A^{\prime \prime}(z) \triangleq A(z)-(A(z) \cdot \nu(z)) \nu(z)$ where $\nu(z)$ denotes a unit normal vector of $\partial \Omega$ at $z$.

Proof of Proposition 6.4. Without loss of generality, we can assume that $\beta \geq 1$. By density argument on $W^{1, p}(\Omega, \mathbb{C})$, we can assume that $u \in C^{\infty}(\bar{\Omega})$. We first observe that, by the classical trace theory and the diamagnetic inequality,

$$
\int_{\partial \Omega}|u|^{p} \leq C_{1}\left(\int_{\Omega}\left|\nabla_{A} U\right|^{p}+|U|^{p}\right)^{\frac{1}{p}}\left(\int_{\Omega}|U|^{p}\right)^{1-1 / p} .
$$

Since $\partial \Omega$ is compact and $\Omega$ is of class $C^{1}$, there exists maps $\psi_{j}: B_{1} \times(-1,1) \subset \mathbb{R}^{d} \times$ $(-1,1) \rightarrow \mathbb{R}^{d+1}$ that are diffeomorphism on their image such that $\psi_{j}\left(B_{1} \times(0,1)\right)=$ $\psi_{j}\left(B_{1} \times(-1,1)\right) \cap \Omega, \psi_{j}\left(B_{1} \times\{0\}\right)=\psi\left(B_{1} \times(-1,1)\right) \cap \partial \Omega, \partial \Omega \subset \bigcup_{j=1}^{\ell} \psi_{j}\left(B_{1 / 2} \times\{0\}\right)$ and for every $j \in\{1, \ldots, \ell\}, \psi_{j}\left(B_{1} \times\{0\}\right)$ is geodesically convex. Applying Proposition 2.4 for $j \in\{1, \ldots, \ell\}$, we have

$$
\begin{aligned}
\iint_{B_{1} \times B_{1}} \frac{\left|e^{i \mathcal{I}_{\psi_{j}^{*} A^{\prime \prime}}(x, y)} u\left(\psi_{j}(y, 0)\right)-u\left(\psi_{j}(x, 0)\right)\right|^{p}}{|y-x|^{d+p-1}} \mathrm{~d} x \mathrm{~d} y \\
\leq C_{2} \iiint_{B_{1} \times[0,1]}\left|\nabla_{\psi_{j}^{*} A}\left(U \circ \psi_{j}\right)\right|^{p}+\beta^{\frac{p}{2}}\left|U \circ \psi_{j}\right|^{p} .
\end{aligned}
$$

Since $\beta \geq 1$, in view of Lemma 6.1 and Lemma 6.2 , this implies that 
$\psi_{j}\left(B_{1} \times\{0\}\right) \times \psi_{j}\left(B_{1} \times\{0\}\right)$

$$
\iint \frac{\left|e^{i \mathcal{I}_{A^{\prime \prime}}^{\partial \Omega}(x, y)} u(y)-u(x)\right|^{p}}{d_{\partial \Omega}(y, x)^{d+p-1}} \mathrm{~d} x \mathrm{~d} y
$$

$$
\leq C_{3}\left(\int_{\psi_{j}\left(B_{1} \times(0,1)\right)}\left|\nabla_{A} U\right|^{p}+\beta^{\frac{p}{2}}|U|^{p}+\beta^{\frac{p-1}{3}} \int_{\psi_{j}\left(B_{1} \times\{0\}\right)}|u|^{p}\right) .
$$

We have then by Young's inequality and by (6.5)

$$
\begin{aligned}
\beta^{\frac{p-1}{3}} \int_{\psi_{j}\left(B_{1} \times\{0\}\right)}|u|^{p} & \leq \beta^{\frac{p-1}{2}} \int_{\psi_{j}\left(B_{1} \times\{0\}\right)}|u|^{p} \\
& \leq C_{4} \int_{\psi_{j}\left(B_{1} \times(0,1)\right)}\left|\nabla_{A} U\right|^{p}+\left(1+\beta^{\frac{p}{2}}\right)|U|^{p} .
\end{aligned}
$$

The conclusion follows by summing for $j \in\{1, \ldots, \ell\}$ the estimates resulting the combination of (6.6) and (6.7).

Concerning the extension, we have

Proposition 6.5. Let $d \geq 1,1 \leq p<+\infty$, and let $\Omega \subset \mathbb{R}^{d+1}$ be a bounded domain of class $C^{1}$. There exists a constant $C_{\Omega, p}>0$ such that if $A \in C^{1}\left(\bar{\Omega}, \bigwedge^{1} \mathbb{R}^{d+1}\right)$ with $\|d A\|_{L^{\infty}(\Omega)} \leq \beta$ and if $u \in W^{1-1 / p, p}(\partial \Omega)$, then there exists $U \in W^{1, p}(\Omega) \cap C^{1}(\Omega, \mathbb{C})$ such that $\operatorname{Tr}_{\partial \Omega} U=u$ and

$$
\int_{\Omega}\left|\nabla_{A} U\right|^{p} \leq C_{\Omega, p}\left(\int_{\substack{(x, y) \in \partial \Omega \times \partial \Omega \\ d_{\partial \Omega}(y, x)<\operatorname{inj}_{\partial \Omega}}} \frac{\left|e^{i \mathcal{I}_{A}^{\partial \Omega}(x, y)} u(y)-u(x)\right|^{p}}{|y-x|^{d+p-1}} \mathrm{~d} x \mathrm{~d} y+\left(1+\beta^{\frac{p-1}{2}}\right) \int_{\partial \Omega}|u|^{p}\right)
$$

and

$$
\int_{\Omega}|U|^{p} \leq \frac{C_{\Omega, p}}{1+\beta^{\frac{p}{2}}} \int_{\partial \Omega}|u|^{p}
$$

Proof. Since $\partial \Omega$ is compact and $\Omega$ is of class $C^{1}$, there exists maps $\psi_{j}: B_{1} \times(-1,1) \subset$ $\mathbb{R}^{d} \times(-1,1) \rightarrow \mathbb{R}^{d}$ that are diffeomorphism on their image which is geodesically convex such that $\psi_{j}\left(B_{1} \times(0,1)\right)=\psi_{j}\left(B_{1} \times(-1,1)\right) \cap \Omega, \psi_{j}\left(B_{1} \times\{0\}\right)=\psi_{j}\left(B_{1} \times(-1,1)\right) \cap \partial \Omega$ and $\partial \Omega \subset \bigcup_{j=1}^{\ell} \psi_{j}\left(B_{1 / 2} \times\{0\}\right)$. Moreover, there exist smooth functions $\eta_{1}, \ldots, \eta_{\ell}$ in $C^{\infty}(\bar{\Omega})$ such that $\operatorname{supp} \eta_{j} \subset \psi_{j}\left(B_{1 / 2} \times[0,1 / 2]\right)$ and $\sum_{j=1}^{\ell} \eta_{j}=1$ in $\partial \Omega$.

By Proposition 3.2, for every $j \in\{1, \ldots, \ell\}$, there exists a function $U_{j} \in W_{\psi_{j}^{*} A}^{1, p}\left(B_{1 / 2} \times\right.$ $[0,1 / 2]) \cap C^{\infty}\left(B_{1 / 2} \times[0,1 / 2]\right)$ such that $\operatorname{Tr} U_{j}=u \circ \psi_{j}$ on $B_{1 / 2} \times\{0\}$ and moreover, 


$$
\begin{array}{r}
\iint_{B_{1 / 2} \times[0,1 / 2]}\left|\nabla_{\psi_{j}^{*} A} U_{j}\right|^{p} \leq C_{1} \iint_{B_{1} \times B_{1}} \frac{\left|e^{i \mathcal{I}_{\psi_{j}^{*} A^{\prime \prime}}(x, y)} u\left(\psi_{j}(y, 0)\right)-u\left(\psi_{j}(x, 0)\right)\right|^{p}}{|y-x|^{d+p-1}} \mathrm{~d} x \mathrm{~d} y \\
+C_{2} \beta^{\frac{p-1}{2}} \int_{B_{1}}\left|u\left(\psi_{j}(x, 0)\right)\right|^{p} \mathrm{~d} x
\end{array}
$$

and (since without loss of generality we may assume that $\beta \geq 1$ )

$$
\iint_{B_{1 / 2} \times[0,1 / 2]}\left|U_{j}\right|^{p} \leq C_{3} \int_{B_{1}}\left|u \circ \psi_{j}\right|^{p} .
$$

Using Lemma 6.1 and Lemma 6.2, we derive that

$$
\begin{gathered}
\iint_{\psi_{j}\left(B_{1 / 2} \times[0,1 / 2]\right)}\left|\nabla_{A}\left(U_{j} \circ \psi_{j}^{-1}\right)\right|^{p} \\
\leq C_{4} \iiint_{\psi_{j}\left(B_{1}\right) \times \psi_{j}\left(B_{1}\right)} \frac{\left|e^{i \mathcal{I}_{A^{\prime \prime}}^{\partial \Omega}(x, y)} u(y)-u(x)\right|^{p}}{|y-x|^{d+p-1}} \mathrm{~d} x \mathrm{~d} y+C_{5} \beta^{\frac{p-1}{2}} \underset{\psi_{j}\left(B_{1} \times\{0\}\right)}{\int}|u|^{p}
\end{gathered}
$$

and

$$
\iint_{\psi_{j}\left(B_{1 / 2} \times[0,1 / 2]\right)}\left|U_{j}\right|^{p} \leq C_{6} \int_{\psi_{j}\left(B_{1} \times\{0\}\right)}|u|^{p} .
$$

We define now $U \triangleq \sum_{j=1}^{\ell} \eta_{j} U_{j} \circ \psi_{j}^{-1}$. We have by the Leibniz rule for covariant derivatives

$$
\nabla_{A} U=\sum_{j=1}^{\ell}\left(\left(U_{j} \circ \psi_{j}^{-1}\right) D \eta_{j}+\eta_{j} \nabla_{A}\left(U_{j} \circ \psi_{j}^{-1}\right)\right),
$$

and we conclude.

Proposition 6.4 and Proposition 6.5 imply the following semi-classical estimates.

Proposition 6.6. For every $A \in C^{1}\left(\bar{\Omega}, \bigwedge^{1} \mathbb{R}^{d+1}\right)$, there exists a positive constant $C$ such that for every $\varepsilon>0$, we have

$$
C^{-1}\left(\underset{\substack{(x, y) \in \partial \Omega \times \partial \Omega \\ d_{\partial \Omega}(y, x)<\operatorname{inj}_{\partial \Omega}}}{\int \varepsilon^{p}\left|e^{i \mathcal{I}_{A}^{\partial \Omega}(x, y) / \varepsilon} u(y)-u(x)\right|^{p}} \frac{1 y-\left.x\right|^{d+p-1}}{\mid y} \mathrm{~d} y+\left(\varepsilon^{p}+\varepsilon^{\frac{p+1}{2}}\right) \int_{\partial \Omega}|u|^{p}\right)
$$




$$
\begin{aligned}
& \leq \inf \left\{\int_{\Omega}|\varepsilon \nabla U+i A U|^{p}+\left(\varepsilon^{p}+\varepsilon^{\frac{p}{2}}\right)|U|^{p} ; U \in W_{A}^{1, p}(\Omega) \text { and } \operatorname{Tr}_{\partial \Omega} U=u\right\} \\
& \leq C\left(\underset{\substack{(x, y) \in \partial \Omega \times \partial \Omega \\
d_{\partial \Omega}(y, x)<\operatorname{inj}_{\partial \Omega}}}{\int} \frac{\varepsilon^{p}\left|e^{i \mathcal{I}_{A}^{\partial \Omega}(x, y) / \varepsilon} u(y)-u(x)\right|^{p}}{|y-x|^{d+p-1}} \mathrm{~d} x \mathrm{~d} y+\left(\varepsilon^{p}+\varepsilon^{\frac{p+1}{2}}\right) \int|u|^{p}\right) . \\
& \partial \Omega \\
&
\end{aligned}
$$

Proof. We define the magnetic potential $A_{\varepsilon} \triangleq A / \varepsilon$, and we observe that $\mathcal{I}_{A_{\varepsilon}}^{\partial \Omega}=\mathcal{I}_{A}^{\partial \Omega} / \varepsilon$, so that

$$
\begin{aligned}
& \iint_{\substack{(x, y) \in \partial \Omega \times \partial \Omega \\
d_{\partial \Omega}(y, x)<\operatorname{inj}_{\partial \Omega}}} \frac{\varepsilon^{p}\left|e^{i \mathcal{I}_{A_{\varepsilon}}^{\partial \Omega}(x, y)} u(y)-u(x)\right|^{p}}{|y-x|^{d+p-1}} \mathrm{~d} x \mathrm{~d} y \\
& =\int_{\substack{(x, y) \in \partial \Omega \times \partial \Omega \\
d_{\partial \Omega}(y, x)<\operatorname{inj}_{\partial \Omega}}} \frac{\varepsilon^{p}\left|e^{i \mathcal{I}_{A}^{\partial \Omega}(x, y) / \varepsilon} u(y)-u(x)\right|^{p}}{|y-x|^{d+p-1}} \mathrm{~d} x \mathrm{~d} y .
\end{aligned}
$$

Moreover, we have

$$
\int_{\Omega}\left|\nabla_{A_{\varepsilon}} U\right|^{p}=\frac{1}{\varepsilon^{p}} \int_{\Omega}|\varepsilon \nabla U+i A U|^{p}
$$

and $\left\|d A_{\varepsilon}\right\|_{L^{\infty}(\Omega)}=\|d A\|_{L^{\infty}(\Omega)} / \varepsilon$. The conclusion follows.

\section{Interpolation of magnetic spaces}

We define for every $p \in[1,+\infty)$ and $\gamma \in(0,+\infty)$, the functional space [34, Definition $1.8 .1 / 1]$

$$
\mathfrak{W}_{A, \gamma}^{1, p}\left(\mathbb{R}^{d}\right)=\left\{U:(0,+\infty) \rightarrow\left(W_{A}^{1, p}\left(\mathbb{R}^{d}\right)+L^{p}\left(\mathbb{R}^{d}\right)\right)\right.
$$

$U$ is weakly differentiable and $\left.\|U\|_{\mathfrak{W}_{A, \gamma}^{1, p}\left(\mathbb{R}^{d}\right)}<+\infty\right\}$,

where

$$
\|U\|_{\mathfrak{W}_{A, \gamma}^{1, p}\left(\mathbb{R}^{d}\right)} \triangleq\left(\int_{0}^{+\infty}\left(\|U(t)\|_{W_{A}^{1, p}\left(\mathbb{R}^{d}\right)}^{p}+\left\|U^{\prime}(t)\right\|_{L^{p}\left(\mathbb{R}^{d}\right)}^{p}\right) t^{\gamma} \mathrm{d} t\right) .
$$

For every $T \in(0,+\infty)$ and $U \in \mathfrak{W}_{A, \gamma}^{1, p}\left(\mathbb{R}^{d}\right)$, one has $U \in C\left([0, T], W_{A}^{1, p}\left(\mathbb{R}^{d}\right)+L^{p}\left(\mathbb{R}^{d}\right)\right)$ [34, Lemma 1.8.1]. In particular, the corresponding trace space can be defined by [34, Definition $1.8 .1 / 2]$ 


$$
\mathfrak{T}_{A, \gamma}^{1, p} \triangleq\left\{U(0) ; U \in \mathfrak{W}_{A, \gamma}^{1, p}\left(\mathbb{R}^{d}\right)\right\}
$$

By a classical result in interpolation theory (see for example [34, Theorem 1.8.2]), we have if $p \in[1,+\infty)$ and $s \in(0,1)$,

$$
\mathfrak{T}_{A,(1-s) p-1}^{1, p}\left(\mathbb{R}^{d}\right)=\left(L^{p}\left(\mathbb{R}^{d}\right), W_{A}^{1, p}\left(\mathbb{R}^{d}\right)\right)_{s, p},
$$

where the right-hand side denotes the real interpolation of order $s$ and exponent $p$ between the spaces $W_{A}^{1, p}\left(\mathbb{R}^{d}\right)$ and $L^{p}\left(\mathbb{R}^{d}\right)$ [34, Definition 1.3.2].

In order to characterize the trace space, we rely on the following equivalence, whose non-magnetic counterpart is classical [34, Lemma 2.9.1/2]

Lemma 7.1. Let $d \geq 1,0<s<1$ and $1 \leq p<+\infty$. If $A \in C\left(\mathbb{R}^{d}, \mathbb{R}^{d}\right)$ and if $d A \in$ $L^{\infty}\left(\mathbb{R}^{d}, \bigwedge^{2} \mathbb{R}^{d}\right)$, then

$$
\mathfrak{W}_{A, \gamma}^{1, p}\left(\mathbb{R}^{d}\right)=W_{\bar{A}, \gamma}^{1, p}\left(\mathbb{R}_{+}^{d+1}\right)
$$

Here $\bar{A}: \mathbb{R}_{+}^{d+1} \rightarrow \mathbb{R}^{d+1}$ is the natural extension of $A$, defined by $\bar{A}(x, t)=(A(x), 0)$. The equality of Lemma 7.1 is understood under the identification $U(t)(x)=U(x, t)$.

Proof of Lemma 7.1. We assume first that $U \in W_{\bar{A}, s}^{1, p}\left(\mathbb{R}_{+}^{d+1}\right)$. By Fubini's theorem, we have for almost every $t, U(t) \in W^{1, p}\left(\mathbb{R}^{d}\right)$. If now $\theta \in C_{c}^{\infty}((0,+\infty))$, we have for every $\varphi \in C_{c}^{\infty}\left(\mathbb{R}^{d}\right)$

$$
\int_{\mathbb{R}^{d}} \int_{0}^{+\infty} \theta^{\prime} \varphi U=-\int_{\mathbb{R}^{d}} \int_{0}^{+\infty} \theta \varphi U^{\prime}
$$

and thus, in $W_{A}^{1, p}\left(\mathbb{R}^{d}\right)+L^{p}\left(\mathbb{R}^{d}\right)$,

$$
\int_{0}^{+\infty} \theta^{\prime}(t) \varphi U(t) \mathrm{d} t=-\int_{0}^{+\infty} \theta(t) \varphi U^{\prime}(t) \mathrm{d} t
$$

We finally have

$$
\int_{0}^{+\infty}\left(\|U(t)\|_{W_{A}^{1, p}\left(\mathbb{R}^{d}\right)}^{p}+\left\|U^{\prime}(t)\right\|_{L^{p}\left(\mathbb{R}^{d}\right)}^{p}\right) t^{\gamma} \mathrm{d} t \leq C_{1} \iint_{\mathbb{R}_{+}^{d+1}}\left(\left|\nabla_{\bar{A}} U(x, t)\right|^{p}+|U(x, t)|^{p}\right) t^{\gamma} \mathrm{d} t \mathrm{~d} x .
$$

Conversely, if $U \in \mathfrak{W}_{A,(1-s) p-1}^{1, p}$, then (7.3) holds and similarly,

$$
\int_{0}^{+\infty} \theta(\operatorname{div} \varphi-i A \cdot \varphi) U(t)=-\int_{0}^{+\infty} \theta \varphi \nabla_{A} U(t)
$$


Hence, by the density of tensor products, we obtain that $U \in W_{\text {loc }}^{1,1}\left(\mathbb{R}_{+}^{d+1}\right)$ and $\nabla_{A} U(t, x)=\left(\nabla_{A}(U(t))(x), U^{\prime}(t)\right)$.

We obtain from the previous results the following characterization of the spaces by interpolation.

Theorem 7.2. Let $d \geq 1,0<s<1$ and $1 \leq p<+\infty$. If $A \in C\left(\mathbb{R}^{d}, \mathbb{R}^{d}\right)$ and if $d A \in L^{\infty}\left(\mathbb{R}^{d}, \bigwedge^{2} \mathbb{R}^{d}\right)$, then

$$
W_{A}^{s, p}=\left(L^{p}\left(\mathbb{R}^{d}\right), W_{A}^{1, p}\left(\mathbb{R}^{d}\right)\right)_{s, p}
$$

Proof. By Theorem 4.3, $W_{A}^{s, p}\left(\mathbb{R}^{d}\right)$ is the trace space of $W_{\bar{A},(1-s) p-1}^{1, p}\left(\mathbb{R}_{+}^{d+1}\right)$. By Lemma 7.1, this latter space coincides with $\mathfrak{W}_{A,(1-s) p-1}^{1, p}\left(\mathbb{R}^{d}\right)$ whose trace space $\mathfrak{T}_{A}^{s, p}\left(\mathbb{R}^{d}\right)$ defined in (7.1) is the required interpolation space by (7.2).

\section{Extension from a half-space}

Finally, we obtain a result about the extension from half-space to the whole space of functions in magnetic Sobolev spaces. Set, for $\gamma \in \mathbb{R}$,

$$
W_{A, \gamma}^{1, p}\left(\mathbb{R}^{d+1}\right) \triangleq\left\{U \in W_{\mathrm{loc}}^{1,1}\left(\mathbb{R}^{d+1}\right) ;\|U\|_{W_{A, \gamma}^{1, p}\left(\mathbb{R}^{d+1}\right)}<+\infty\right\}
$$

where

$$
\|U\|_{W_{A, \gamma}^{1, p}\left(\mathbb{R}^{d+1}\right)} \triangleq\left(\iint_{\mathbb{R}_{+}^{d+1}}\left(\left|\nabla_{A} U(x, t)\right|^{p}+|U(x, t)|^{p}\right)|t|^{\gamma} \mathrm{d} x \mathrm{~d} t\right)^{\frac{1}{p}}
$$

Theorem 8.1. Let $d \geq 1,-1<\gamma<p-1$ and $1 \leq p<+\infty$. There exists a constant $C>0$ such that for every $A \in C^{1}\left(\mathbb{R}^{d+1}, \bigwedge^{1} \mathbb{R}^{d+1}\right)$ such that $d A$ is bounded and every $U \in W_{A, \gamma}^{1, p}\left(\mathbb{R}_{+}^{d+1}\right)$, there exists $\bar{U} \in W_{A, \gamma}^{1, p}\left(\mathbb{R}^{d+1}\right)$ such that $\bar{U}=U$ on $\mathbb{R}_{+}^{d+1}$. Moreover, if $\beta \geq\|d A\|_{L^{\infty}\left(\mathbb{R}^{d+1}\right)}$,

$$
\iint_{\mathbb{R}^{d+1}}\left(\left|\nabla_{A} \bar{U}(x, t)\right|^{p}+\beta^{\frac{p}{2}}|\bar{U}(x, t)|^{p}\right) t^{\gamma} \mathrm{d} x \mathrm{~d} t \leq C \iint_{\mathbb{R}^{d+1}}\left(\left|\nabla_{A} U(x, t)\right|^{p}+\beta^{\frac{p}{2}}|U(x, t)|^{p}\right) t^{\gamma} \mathrm{d} x \mathrm{~d} t .
$$

Proof of Theorem 8.1. This follows from Theorem 4.3 on $\mathbb{R}_{+}^{d+1}$ and Proposition 3.1 on $\mathbb{R}_{-}^{d+1}$ with $s=1-\frac{\gamma+1}{p}$.

Remark 8.2. A natural strategy to prove Theorem 8.1 would be to define the extension $\bar{U}$ by reflection: for every $(x, t) \in \mathbb{R}^{d} \times(-\infty, 0)$, we would define $\bar{U}(x, t)=U(x,-t)$. The 
computation of the covariant derivative would give

$$
\nabla_{A} \bar{U}(x, t)=R \nabla_{A} U(x,-t)+i(A(x, t)-R A(x-t)) U(x,-t)
$$

where $R$ is the orthogonal reflection with respect to the hyperplane $\mathbb{R}^{d} \times\{0\}$. The approach would thus only work when $A$ is invariant under the pull-back by $R$; this would imply the same invariance of the magnetic field $d A$ and implies that even up to gauge transformations, it is not possible to cover a significant class of magnetic fields.

\section{Appendix A. Alternative magnetic Gagliardo seminorms}

A fractional Gagliardo seminorm defined by an integral involving the mid-point has been proposed in the literature (see $[1,2,5, \S 2]$, [8, §2], [12,15,18, §2], [25,27,28,33,37, $\S 2])$ :

$$
\left(\iint_{\mathbb{R}^{d} \times \mathbb{R}^{d}} \frac{\left|e^{i A\left(\frac{x+y}{2}\right)[y-x]} u(y)-u(x)\right|^{p}}{|y-x|^{d+s p}} \mathrm{~d} x \mathrm{~d} y\right)^{\frac{1}{p}}
$$

another natural candidate could be the integral involving boundary points

$$
\left(\iint_{\mathbb{R}^{d} \times \mathbb{R}^{d}} \frac{\left|e^{\frac{i}{2}(A(x)[y-x]+A(y)[y-x])} u(y)-u(x)\right|^{p}}{|y-x|^{d+s p}} \mathrm{~d} x \mathrm{~d} y\right)^{\frac{1}{p}} .
$$

Whereas (1.4) enjoyed a gauge-invariance property, this is not the case for (A.1) or (A.2).

The formulas (1.4), (A.1) and (A.2) are in fact particular cases of the following general semi-norm

$$
\|u\|_{\dot{W}_{A, \mu}^{s, p}\left(\mathbb{R}^{d}\right)} \triangleq\left(\iint_{\mathbb{R}^{d} \times \mathbb{R}^{d}} \frac{\left|e^{i \mathcal{I}_{A}^{\mu}(x, y)} u(y)-u(x)\right|^{p}}{|y-x|^{d+s p}} \mathrm{~d} x \mathrm{~d} y\right)^{\frac{1}{p}}
$$

where $\mu$ is a given finite measure on the interval $[0,1]$ and where the potential $\mathcal{I}_{A}^{\mu}$ of $A$ with respect to the measure $\mu$ is defined by the following variant of (2.4)

$$
\mathcal{I}_{A}^{\mu}(x, y) \triangleq \int_{0}^{1} A((1-t) x+t y) \cdot(y-x) \mathrm{d} \mu(t) .
$$

The seminorm defined in (1.4), (A.1) and (A.2) correspond respectively to a restriction of the Lebesgue measure $\mu=\mathcal{L}^{1}\left\llcorner[0,1]\right.$, a Dirac measure at the center $\mu=\delta_{1 / 2}$ and the average of Dirac measures at the endpoint $\mu=\frac{\delta_{0}+\delta_{1}}{2}$. 
Proposition A.1. Let $k \in \mathbb{N}$ and let $\mu_{1}, \mu_{2}$ be measures on $[0,1]$. If for every $j \in$ $\{0, \ldots, k-1\}$

$$
\int_{0}^{1} t^{j} \mathrm{~d} \mu_{1}(t)=\int_{0}^{1} t^{j} \mathrm{~d} \mu_{2}(t),
$$

then

$$
\left|\|u\|_{W_{A, \mu_{2}}^{s, p}\left(\mathbb{R}^{d}\right)}-\|u\|_{W_{A, \mu_{1}}^{s, p}\left(\mathbb{R}^{d}\right)}\right| \leq C\left\|D^{k} A\right\|_{L^{\infty}\left(\mathbb{R}^{d}\right)}^{\frac{s}{k+1}}\|u\|_{L^{p}\left(\mathbb{R}^{d}\right)} .
$$

The measures corresponding to the semi-norms of (1.4), (A.1) and (A.2) all satisfy the assumptions of Proposition A.1 with $k=0, k=1$ and $k=2$, and the heterogeneous spaces coincide thus as soon as either $A$ is bounded or its second or first derivative is bounded. This means in particular that if $k=1$, the estimates in the present work involving $\|d A\|_{L^{\infty}}$ and our semi-norm given by (1.4), have counterparts involving $\|D A\|_{L^{\infty}}$ and either (A.1) and (A.2); the latter quantities are not gauge-invariant.

In the particular case where $A$ is an affine function, then Proposition A.1 holds with $k=2$ and $D^{2} A=0$; the norms defined by (1.4), (A.1) and (A.2) are then identical.

Higher moment identities in the assumption of Proposition A.1 induce lower powers in the dependence on derivatives of $A$, which can be relevant in semi-classical analyses. The exponent in the moment condition can be increased by using a measure $\mu$ such that more moment coincide. For instance setting $\mu=\frac{1}{6} \delta_{0}+\frac{2}{3} \delta_{1 / 2}+\frac{1}{6} \delta_{1}$, corresponding to Simpson's quadrature rule, would give estimates of (1.4) up to a term of the order $\left\|D^{k} A\right\|_{L^{\infty}\left(\mathbb{R}^{d}\right)}^{\frac{s p}{k+1}}$ for $k \in\{0, \ldots, 3\}$.

Proof of Proposition A.1. We have for every $x, y \in \mathbb{R}^{d}$, by the triangle inequality

$$
\begin{aligned}
|| e^{i \mathcal{I}_{A}^{\mu_{2}}(x, y)} u(y)-u(x)|-| e^{i \mathcal{I}_{A}^{\mu_{1}}(x, y)} u(y) & -u(x)|| \\
& \leq\left|e^{i\left(\mathcal{I}_{A}^{\mu_{2}}(x, y)-\mathcal{I}_{A}^{\mu_{2}}(x, y)\right.} u(y)\right|=\left|e^{i \mathcal{I}_{A}^{\mu_{2}-\mu_{1}}(x, y)} u(y)\right| .
\end{aligned}
$$

By integration and Minkowski's inequality, this yields

$$
\begin{gathered}
\mid\left(\iint_{\mathbb{R}^{d} \times \mathbb{R}^{d}} \frac{\left|e^{i \mathcal{I}_{A}^{\mu_{2}}(x, y)} u(y)-u(x)\right|^{p}}{|y-x|^{d+s p}} \mathrm{~d} x \mathrm{~d} y\right)^{\frac{1}{p}} \\
\quad-\left(\iint_{\mathbb{R}^{d} \times \mathbb{R}^{d}} \frac{\left|e^{i \mathcal{I}_{A}^{\mu_{1}}(x, y)} u(y)-u(x)\right|^{p}}{|y-x|^{d+s p}} \mathrm{~d} x \mathrm{~d} y\right)^{\frac{1}{p} \mid} \\
\leq\left(\iint_{\mathbb{R}^{d} \times \mathbb{R}^{d}} \frac{\left|e^{i \mathcal{I}_{A}^{\mu_{2}-\mu_{1}}(x, y)}-1\right|^{p}|u(y)|^{p}}{|y-x|^{d+s p}} \mathrm{~d} x \mathrm{~d} y\right)^{\frac{1}{p}} .
\end{gathered}
$$


By our assumption on the moments of the measures $\mu_{1}$ and $\mu_{2}$, there exists a constant $C_{1}$ depending only on the measure $\mu_{2}-\mu_{1}$ such that

$$
\left|\mathcal{I}_{A}^{\mu_{2}-\mu_{1}}(x, y)\right| \leq C_{1}\left\|D^{k} A\right\|_{L^{\infty}\left(\mathbb{R}^{d}\right)}|y-x|^{k+1}
$$

and thus for every $y \in \mathbb{R}^{d}$

$$
\int_{\mathbb{R}^{d}} \frac{\left|e^{i \mathcal{I}_{A}^{\mu_{2}-\mu_{1}}(x, y)}-1\right|^{p}}{|y-x|^{d+s p}} \mathrm{~d} x \leq C_{2}\left\|D^{k} A\right\|_{L^{\infty}\left(\mathbb{R}^{d}\right)}^{\frac{s p}{k+1}}
$$

the conclusion then follows.

\section{References}

[1] V. Ambrosio, Boundedness and decay of solutions for some fractional magnetic Schrödinger equations in $\mathbb{R}^{N}$, Milan J. Math. 85 (1) (2018) 127-150, https://doi.org/10.1007/s00032-018-0283-3.

[2] V. Ambrosio, P. d'Avenia, Nonlinear fractional magnetic Schrödinger equation: existence and multiplicity, J. Differ. Equ. 264 (5) (2018) 3336-3368, https://doi.org/10.1016/j.jde.2017.11.021.

[3] G. Arioli, A. Szulkin, A semilinear Schrödinger equation in the presence of a magnetic field, Arch. Ration. Mech. Anal. 170 (4) (2003) 277-295, https://doi.org/10.1007/s00205-003-0274-5.

[4] J. Avron, I. Herbst, B. Simon, Schrödinger operators with magnetic fields, I: general interactions, Duke Math. J. 45 (4) (1978) 847-883.

[5] Z. Binlin, M. Squassina, Z. Xia, Fractional NLS equations with magnetic field, critical frequency and critical growth, Manuscr. Math. 155 (1-2) (2018) 115-140, https://doi.org/10.1007/s00229-0170937-4.

[6] S. Cingolani, S. Secchi, Semiclassical limit for nonlinear Schrödinger equations with electromagnetic fields, J. Math. Anal. Appl. 275 (1) (2002) 108-130, https://doi.org/10.1016/S0022-247X(02)002780 .

[7] R.R. Coifman, C. Fefferman, Weighted norm inequalities for maximal functions and singular integrals, Stud. Math. 51 (1974) 241-250, https://doi.org/10.4064/sm-51-3-241-250.

[8] P. d'Avenia, M. Squassina, Ground states for fractional magnetic operators, ESAIM Control Optim. Calc. Var. 24 (1) (2018) 1-24, https://doi.org/10.1051/cocv/2016071.

[9] E. DiBenedetto, Real Analysis, 2nd ed., Birkhäuser Advanced Texts: Basler Lehrbücher, Birkhäuser/Springer, New York, 2016.

[10] M.P. do Carmo, Riemannian Geometry, Mathematics: Theory \& Applications, Birkhäuser, Boston, MA, 1992.

[11] M.J. Esteban, P.-L. Lions, Stationary solutions of nonlinear Schrödinger equations with an external magnetic field, in: Partial Differential Equations and the Calculus of Variations, Vol. I, in: Progr. Nonlinear Differential Equations Appl., vol. 1, Birkhäuser, Boston, MA, 1989, pp. 401-449.

[12] A. Fiscella, A. Pinamonti, E. Vecchi, Multiplicity results for magnetic fractional problems, J. Differ. Equ. 263 (8) (2017) 4617-4633, https://doi.org/10.1016/j.jde.2017.05.028.

[13] E. Gagliardo, Caratterizzazioni delle tracce sulla frontiera relative ad alcune classi di funzioni in $n$ variabili, Rend. Semin. Mat. Univ. Padova 27 (1957) 284-305.

[14] S. Gasiorowicz, Quantum Physics, 3rd ed., Wiley, Hoboken, N.J., 2003.

[15] T. Ichinose, Magnetic relativistic Schrödinger operators and imaginary-time path integrals, in: Mathematical Physics, Spectral Theory and Stochastic Analysis, in: Oper. Theory Adv. Appl., vol. 232, Birkhäuser/Springer, Basel, 2013, pp. 247-297.

[16] T. Kato, Schrödinger operators with singular potentials, in: Proceedings of the International Symposium on Partial Differential Equations and the Geometry of Normed Linear Spaces (Jerusalem, 1972), Isr. J. Math. 13 (1972) 135-148, https://doi.org/10.1007/BF02760233 (1973).

[17] L.D. Landau, E.M. Liftschitz, Quantum Mechanics: Non-Relativistic Theory, 3rd ed., Course of Theoretical Physics, vol. 3, Pergamon Press, Oxford, 1977. 
[18] S. Liang, D. Repovš, B. Zhang, On the fractional Schrödinger-Kirchhoff equations with electromagnetic fields and critical nonlinearity, Comput. Math. Appl. 75 (5) (2018) 1778-1794, https:// doi.org/10.1016/j.camwa.2017.11.033.

[19] E.H. Lieb, M. Loss, Analysis, 2nd ed., Graduate Studies in Mathematics, vol. 14, American Mathematical Society, Providence, RI, 2001, xxii+346.

[20] J.-L. Lions, J. Peetre, Sur une classe d'espaces d'interpolation, Publ. Math. IHÉS (19) (1964) 5-68.

[21] N. Miller, Weighted Sobolev spaces and pseudodifferential operators with smooth symbols, Trans. Am. Math. Soc. 269 (1) (1982) 91-109, https://doi.org/10.2307/1998595.

[22] P. Mironescu, E. Russ, Traces of weighted Sobolev spaces: old and new, Nonlinear Anal. 119 (2015) 354-381, https://doi.org/10.1016/j.na.2014.10.027.

[23] B. Muckenhoupt, Weighted norm inequalities for the Hardy maximal function, Trans. Am. Math. Soc. 165 (1972) 207-226, https://doi.org/10.2307/1995882.

[24] B. Muckenhoupt, R.L. Wheeden, On the dual of weighted $H^{1}$ of the half-space, Stud. Math. 63 (1) (1978) 57-79, https://doi.org/10.4064/sm-63-1-57-79.

[25] H.-M. Nguyen, A. Pinamonti, M. Squassina, E. Vecchi, New characterizations of magnetic Sobolev spaces, Adv. Nonlinear Anal. 7 (2) (2018) 227-245, https://doi.org/10.1515/anona-2017-0239.

[26] H.-M. Nguyen, M. Squassina, On anisotropic Sobolev spaces, Commun. Contemp. Math. 21 (1) (2019) 1850017, 13 pp.

[27] A. Pinamonti, M. Squassina, E. Vecchi, The Maz'ya-Shaposhnikova limit in the magnetic setting, J. Math. Anal. Appl. 449 (2) (2017) 1152-1159, https://doi.org/10.1016/j.jmaa.2016.12.065.

[28] A. Pinamonti, M. Squassina, E. Vecchi, Magnetic BV-functions and the Bourgain-Brezis-Mironescu formula, Adv. Calc. Var. 12 (3) (2019) 225-252, https://doi.org/10.1515/acv-2017-0019.

[29] N. Raymond, Bound States of the Magnetic Schrödinger Operator, EMS Tracts in Mathematics, vol. 27, European Mathematical Society (EMS), Zürich, 2017.

[30] O.A. Salieva, Direct and converse imbedding theorems for seminormed spaces, Anal. Math. 15 (2) (1989) 145-158, https://doi.org/10.1007/BF01910946.

[31] E. Sandier, S. Serfaty, Vortices in the Magnetic Ginzburg-Landau Model, Progress in Nonlinear Differential Equations and Their Applications, vol. 70, Birkhäuser, Boston, Mass., 2007.

[32] S.B. Sontz, Principal Bundles, The Classical Case, Universitext, Springer, Cham, 2015.

[33] M. Squassina, B. Volzone, Bourgain-Brézis-Mironescu formula for magnetic operators, C. R. Math. Acad. Sci. Paris 354 (8) (2016) 825-831, https://doi.org/10.1016/j.crma.2016.04.013.

[34] H. Triebel, Interpolation Theory, Function Spaces, Differential Operators, North-Holland Mathematical Library, vol. 18, North-Holland, Amsterdam-New York, 1978.

[35] B.O. Turesson, Nonlinear Potential Theory and Weighted Sobolev Spaces, Lecture Notes in Mathematics, vol. 1736, Springer-Verlag, Berlin, 2000.

[36] S.V. Uspenskiǔ, Imbedding theorems for classes with weights, Tr. Mat. Inst. Steklova 60 (1961) 282-303 (Russian).

[37] F. Wang, M. Xiang, Multiplicity of solutions to a nonlocal Choquard equation involving fractional magnetic operators and critical exponent, Electron. J. Differ. Equ. (2016), Paper No. 306, 11.

[38] M. Willem, Functional Analysis: Fundamentals and Applications, Cornerstones, Birkhäuser/ Springer, New York, 2013. 